\title{
15 Juli Zehs Poetik des Dystopischen
}

Man dürfte keinen allzu großen Widerspruch riskieren, indem man Juli Zeh als die prominenteste und mit ihrem öffentlichen Engagement am stärksten wahrgenommene politische Schriftstellerin ihrer Generation bezeichnet. ${ }^{1069}$ (Was die akademische Rezeption angeht, so könnte Zeh allenfalls Kathrin Röggla zur Seite gestellt werden, deren Werk weiter oben diskutiert wurde. ${ }^{1070}$ ) Immer wieder thematisiert Zeh in ihren schriftstellerischen Arbeiten politische, moralische und gesellschaftliche Fragestellungen, etwa die Entwicklung Bosniens nach Ende des Krieges in der Reiseerzählung Die Stille ist ein Geräusch (2002), die Einschränkung bürgerlicher Rechte im Roman Corpus Delicti (2009), die Anwendung von Foltermethoden im Kampf gegen vermeintliche Terroristen im Theaterstück Der Kaktus (2009) oder das Schwinden des öffentlichen Diskurses und den wachsenden Einfluss neurechter Bewegungen im Roman Leere Herzen (2017). Auch in Reden, Interviews und Zeitungsartikeln hat Zeh wiederholt Stellung zu aktuellen politischen und gesellschaftlichen Fragen bezogen, beispielsweise zur Etablierung eines „präventiv denkende[n] und handelnde[n] Kontrollsystem[s] ${ }^{\text {“1071 }}$ bei Krankenkassenregelungen oder zur Datenüberwachung. ${ }^{1072}$ Gelegentlich versucht die Autorin sogar, unmittelbar Einfluss auf die Politik auszuüben: So reichte Zeh im Januar 2008 beim Bundesverfassungsgericht eine - erfolglos gebliebene - Verfassungsbeschwerde gegen die obligatorische Erfassung von Fingerabdrücken auf dem biometrischen Reisepass ein; im Juli 2013 stand Zeh an der Spitze einer Liste von Schriftstellern, die Bundeskanzlerin Angela Merkel in einem offenen Brief dazu aufforderten, „den Menschen im Land die volle Wahrheit über die Spähangriffe“ der NSA mitzuteilen; ${ }^{1073}$ und im Dezember 2018 wurde die promovierte Juristin Zeh zur ehrenamtlichen Richterin

1069 Vgl. Wagner: Aufklärer der Gegenwart, S. 64.

1070 Allerdings unterscheiden sich Ästhetik und politischer Anspruch im Werk Rögglas deutlich von denjenigen im Werk Zehs. In einem Artikel aus dem Jahre 2004 schreibt Zeh, dass sie in den Werken von „Kathrin Röggla, Ingo Schulze und Thomas Meinecke [...] das Politische nur mit Mühe aufspüren kann“ (Juli Zeh: Auf den Barrikaden oder hinter dem Berg. Die jungen Schriftsteller und die Politik. In: Undine Ruge / Daniel Morat (Hg.): Deutschland denken. Beiträge für die reflektierte Republik. Wiesbaden 2005, S. 23-28, hier S. 25). Siehe für einen Vergleich zwischen der politischen Ästhetik Zehs und Rögglas Kapitel 17. Versuch eines politischen Vergleichs: Kracht, Röggla, Zeh, Randt.

1071 Juli Zeh: Patienten. Vom Sozialstaat zum Kontrollsystem. In: Zeit online, 29.07.2010. Quelle: https://www.zeit.de/online/2007/41/meldepflicht-patienten (Zugriff: 27.07.2021).

1072 Juli Zeh: Schützt den Datenkörper. In: Frankfurter Allgemeine Zeitung, 11.02.2014.

1073 Juli Zeh / Ilija Trojanow / Carolin Emcke u. a.: Offener Brief an Angela Merkel. Deutschland ist ein Überwachungsstaat. In: Frankfurter Allgemeine Zeitung, 25.07.2013.

Ә Open Access. (C) 2022 Michael Navratil, publiziert von De Gruyter. (c) BY-NC-ND Dieses Werk ist lizenziert unter einer Creative Commons Namensnennung - Nicht-kommerziell - Keine Bearbeitung 4.0 International Lizenz. https://doi.org/10.1515/9783110763119-018 
am Verfassungsgericht des Landes Brandenburg gewählt. Zehs Einsatz für politische Belange, sowohl im Rahmen ihres literarischen Schaffens als auch darüber hinaus, lässt die Autorin als „Intellektuelle klassischen Musters“"1074 respektive als „public intellectual“ erscheinen. ${ }^{1075}$

Für die Literaturwissenschaft stellt sich damit - wie stets beim Blick auf politische Schriftsteller - die Frage, in welchem Verhältnis politisches Wirken und literarische Produktion im Falle Zehs zueinander stehen. Die Autorin selbst weist in diesem Zusammenhang einerseits auf die grundsätzliche politische Dimension literarischen Schreibens hin: „Politik wird nicht an internationalen Konferenztischen gemacht, sondern zuallererst in den Köpfen der Menschen, in denen sich, ja: Wörter befinden. [...] So begriffen, ist Schreiben immer [...] ein politischer Akt““ ${ }^{1076}$ Andererseits macht Zeh darauf aufmerksam, dass es „nicht [reicht], Ästhet zu sein, wenn man ein moralisches Konzept erschaffen will. Jeder politischen oder moralischen Wirkung muss eine Grundentscheidung vorausgehen: für das, was man will, oder wenigstens gegen das, was man nicht will."1077 Entsprechend warnt Zeh vor einem überinklusiven Begriff des Politischen, der rundweg die gesamte Sinnproduktion fiktionaler Literatur umfasst: „Ich finde [...], man braucht den Begriff des Politischen an der Stelle nicht, weil er sehr beliebig wird, wenn man ihn zu stark ausweitet. Ich würde den Begriff des Politischen für die Literatur enger fassen, und vor allem [...] würde ich ihn immer über die Intention definieren. “1078 Noch deutlicher programmatisch formuliert Zeh in ihren Frankfurter Poetikvorlesungen: „Echte politische Literatur liegt nur dann vor, wenn der Autor beim Schreiben ein konkretes politisches Ziel verfolgte. “1079

1074 Heinz-Peter Preußer: Gewalt und Überwachung. Juli Zehs apokalyptisches Pandämonium der Jetztzeit und ihre düstere Prognose der ,Selbstoptimierung in Corpus Delicti. In: Olaf Briese / Richard Faber / Madleen Podewski (Hg.): Aktualität des Apokalyptischen. Zwischen Kulturkritik und Kulturversprechen. Würzburg 2015, S. 163-185, hier S. 180, Anm. 55.

1075 Vgl. Patricia Herminghouse: The Young Author as Public Intellectual. The Case of Juli Zeh. In: Dies. / Katharina Gerstenberger (Hg.): German Literature in a New Century: Trends, Traditions, Transitions, Transformations. New York 2008, S. 268-284.

1076 Juli Zeh: Nachts sind das Tiere. Frankfurt a. M. 2014, S. 101. Siehe auch Zeh: Auf den Barrikaden oder hinter dem Berg, S. 27.

1077 Juli Zeh: Gesellschaftliche Relevanz braucht eine politische Richtung. In: Die Zeit, 23.06.2005.

1078 Juli Zeh: „Ich weiß, dass ich permanent über Moral schreibe.“ Juli Zeh im Gespräch. In: Stephanie Waldow (Hg.): Ethik im Gespräch. Autorinnen und Autoren über das Verhältnis von Literatur und Ethik heute. Bielefeld 2011, S. 55-64, hier S. 57.

1079 Juli Zeh: Treideln. Frankfurter Poetikvorlesungen. Frankfurt a. M. 2013, S. 133. 
2011 reservierte Zeh in einem Interview einen solch engen Begriff des Politischen innerhalb ihres eigenen Werks für genau zwei Bücher (wobei unter Zehs seither erschienenen Romanen möglicherweise auch noch Leere Herzen zur politischen Literatur gezählt werden könnte):

Wenn ich etwas von „aufrütteln“ gesagt habe und die Leser direkt ansprechen will dann beziehe ich mich auf genau zwei Bücher: nämlich auf Angriff auf die Freiheit mit Ilija Trojanow und auf Corpus Delicti, was eigentlich ein Theaterstück war. Das bezieht sich nicht auf die Romane. [...]

Bei Corpus Delicti und Angriff auf die Freiheit war das wirklich ein ganz altmodisches, aufklärerisches Unterfangen, zu dem ich dann auch stehe - zum erhobenen Zeigefinger, zur Kanzel, zum Essayistischen, Diskurshaften, Thesenhaften. Bei den Romanen ging es hingegen nicht um Aufklärung oder Präsentation einer Haltung. ${ }^{1080}$

Zeh bekennt sich hier, wenn auch lediglich in Bezug auf einige wenige ihrer Bücher, zu einer aufklärerischen Form politischer Literatur, die sich als Anknüpfung an die spezifisch deutschen Tradition der ,engagierten Literatur' begreifen lässt. ${ }^{1081}$ Zugleich zeichnen sich im angeführten Zitat Grundzüge einer Gattungspoetik des Politischen ab: Während sich Essay und Sachbuch qua Gattung gut zur Problematisierung politischer Fragestellungen eigenen, erachtet Zeh den Versuch, politische Romane zu verfassen - literarische Texte also, in denen sich ein künstlerischer Anspruch mit einer klaren politischen Botschaft verbindet -, als tendenziell problematisch. In ihren Frankfurter Poetikvorlesungen formuliert Zeh hierzu: „Es ist gerade die Definition von ernsthafter Literatur, dass der Autor vorne nicht weiß, was beim Lesen hinten rauskommt. Gute Literatur ist Kunst, und Kunst ist kein karitativer, sondern ein narzisstischer Akt. ${ }^{\text {"1082 Corpus }}$ Delicti stellt entsprechend innerhalb von Zehs Werk eine Ausnahme dar, insofern die Autorin hier, in ihren eigenen Worten, „das erste Mal [...] versucht habe, mit einem literarischen Text auch eine ziemlich klare politische Botschaft auszusenden.“1083

Der Roman Corpus Delicti verdankt, so Zehs Sichtweise, die vergleichsweise große Deutlichkeit seiner politischen Botschaft wesentlich dem Umstand, dass

1080 Zeh: „Ich weiß, dass ich permanent über Moral schreibe.“, S. $57 \mathrm{f}$.

1081 Siehe Kapitel 7. Politisches Schreiben: Kein Klärungsversuch.

1082 Zeh: Treideln, S. 32.

1083 Christoph Borgans / Michaela Meißner / Juli Zeh: „Ich bin ein großer Fan der Freiheit“. In: unique. Interkulturelles Studentenmagazin für Jena, Weimar \& Erfurt, 11.05.2011. Quelle: http://www.unique-online.de/\%E2\%80\%9Eich-bin-ein-groser-fan-der-freiheit $\%$ E2\%80\%9C/ 3340/ (Zugriff: 27.07.2021). Im Kommentarband Fragen zu ,Corpus Delicti` von 2020 hat Zeh noch einmal bestätigt, „dass Corpus Delicti eine Sonderposition in meinem gesamten literarischen Werk einnimmt. Es ist mein erster, vielleicht auch mein einziger politischer Roman.“ (Juli Zeh: Fragen zu Corpus Delicti. München 2020, S. 14). 
der Stoff ursprünglich in Form eines Theaterstücks behandelt wurde: Die Uraufführung des Stückes Corpus Delicti fand im September 2007 bei der Ruhrtriennale in Essen statt. In einem Interview, das zwischen der Entstehung der Dramen- und der Romanfassung von Corpus Delicti entstand, weist Zeh darauf hin, dass das Schreiben dieses Dramentextes eine „Extrawurst“ innerhalb ihres schriftstellerischen Schaffens dargestellt habe: „Beim Drama [...] hatte ich den Eindruck, ich kann hier politische Meinungen vertreten, ich kann sagen, was ich will, sogar noch eher als im Essay. “1084 Die Abkunft vom politischen Dramentext ist auch der Romanfassung von Corpus Delicti noch stellenweise anzumerken: in der starken Reduzierung des Personals und dem vergleichsweise großen Anteil dialogischer Passagen ebenso wie in der deutlichen politischen Tendenz. ${ }^{1085}$

Das dominierende Genre in Zehs Werk bildet zweifellos der ,alltagsrealistische[...] Gegenwartsroman“, den Zeh selbst auch über ihr eigenes Schaffen hinaus zur dominierenden literarischen Form der deutschen Gegenwartsliteratur erklärt hat. ${ }^{1086}$ Sondert man allerdings diejenigen literarischen Werke Zehs aus, die sich im engeren Sinne als politisch - im oben erläuterten Verständnis bezeichnen lassen, so fällt auf, dass es sich bei fast allen diesen Werken um Dystopien handelt, also gerade nicht um alltagsrealistische Texte: Neben den Romanen Corpus Delicti und Leere Herzen wäre vor allem das Stück Der Kaktus zu nennen, mit gewissen Einschränkungen auch noch das Stück 203. ${ }^{1087}$ Dass in Zehs Werk ausgerechnet das Zukünftige als präferierte Zeitstufe für die Kommentierung gegenwärtiger politischer Entwicklungen fungiert, mag zunächst überraschen, scheint das futurische Setting der Dystopie doch gerade von der Gegenwart wegzuführen. Tatsächlich weisen Zehs dystopische Texte aber sämtlich eine enge Bindung an die Gegenwart ihrer Entstehung, inklusive ihrer politischen Entwicklungen und gesellschaftlichen Diskurse, auf. So behauptet Zeh im Interview, sie habe mit dem Roman Corpus Delicti „Dinge, die jetzt schon da sind, in ein fiktives System übertragen und ein bisschen über-

1084 Juli Zeh: Der Unterschied zwischen Realität und Fiktion ist marginal. Oldenburg 2008, S. 68.

1085 So hält Inga Ketels für Zehs Roman fest, dass aufgrund seiner „Textgeschichte [...] der dem Genre des dystopischen Romans eigene diskursive Charakter hier besonders stark ausgeprägt [ist]; der als auktoriale Erzählung verfasste Roman beinhaltet zahlreiche Dialoge, in denen das jeweilige Politikverständnis der Figuren dargelegt wird. In diesem Sinne kann der Roman als novel of ideas, beziehungsweise als philosophischer Roman verstanden werden, in dem unterschiedliche moralische Standpunkte gegeneinander antreten. “ (Ketels: Der Einzug des Politischen in die Gegenwartsliteratur, S. 113).

1086 Vgl. Zeh: Der Unterschied zwischen Realität und Fiktion ist marginal, S. 40.

1087 In 203 geht es um die Konstruktion von Realität und Identität in einem totalitären Regime. 
dreht“. ${ }^{1088}$ Offenbar besteht die Funktion des dystopischen Schreibens für Zeh weder in der Ausgestaltung komplexer eigenständiger Welten, wie sie für die Genres der Science-Fiction und der Fantasy charakteristisch sind, noch auch in einer futurologischen Folgenabschätzung oder der Warnung vor einer als wahrscheinlich angenommenen Zukunft. Die besondere Eignung der Dystopie als Form politischen Schreibens ergibt sich für Zeh vielmehr aus der Fähigkeit des Genres, gesellschaftliche und politische Tendenzen der Gegenwart durch Überspitzung, ,Überdrehung، oder eben kontrafaktische Variation in besonderer Deutlichkeit zu konturieren und damit öffentlich zur Diskussion zu stellen. ${ }^{1089}$ Im Interview danach befragt, ob es überhaupt noch einen Sinn habe, Dystopien zu schreiben, wenn sich doch selbst im Falle einer intensiven öffentlichen Rezeption keine gesellschaftlichen Veränderungen einstellten, entgegnet Zeh:

Ich sehe die Funktion von Literatur anders. Es geht in erster Linie darum, dass wir uns als Menschen miteinander verständigen, einen Diskurs führen, in dem bestimmte Themen aufgenommen werden. Daher bin ich der Ansicht, dass Literatur auch ein traditionell aufklärerisches Anliegen ist. Ich betrachte das Nachdenken über Dinge schon als einen sehr wichtigen Wert an sich und ich glaube, dass es definitiv das Bewusstsein von Menschen verändert. Und wir können diese „Was-wäre-wenn-Gleichung“ nicht ernsthaft ziehen: Wir wissen nicht wie die Welt aussähe, wenn 1984 von Orwell nicht erschienen wäre. Ich war mit der Rezeption und der Auswirkung von Corpus Delicti wirklich zufrieden. Es war tatsächlich so, dass ich gemerkt habe, wie viele Menschen dieses Thema schon auf dem Schirm hatten und darüber auch tatsächlich sprechen wollten. Sie hatten bereits darüber nachgedacht und dann ist solch ein Buch ein willkommener Anlass, um die Diskussion wirklich aufzunehmen und damit ist schon viel erreicht. ${ }^{1090}$

Die politische Dimension von Zehs Dystopien liegt demnach nicht in der Forderung nach einem radikalen Bruch im Bereich des Politischen begründet, ja noch nicht einmal in der Hoffnung auf die Einführung eines bisher ignorierten Themas in den öffentlichen Diskurs; vielmehr versucht Zeh, sich mittels ihrer literarischen Texte in ebendiesen öffentlichen Diskurs einzuschreiben. Sie tut dies, indem sie die politische Relevanz bestimmter zeitgenössischer Themen in ihren Dystopien in besonderer Deutlichkeit herausstreicht und damit implizit zu einer verstärkten öffentlichen Diskussion dieser Themen aufruft.

1088 Johannes Gernert / Juli Zeh: Plädoyer gegen die Fitness-Diktatur. In: Stern, 24.03.2009. 1089 Auch im Rahmen ihrer journalistischen Tätigkeit greift Zeh mitunter auf kontrafaktische Argumentationsformen zurück. Siehe Juli Zeh: Alltag ohne. Was wäre heute, wenn der 11. September 2001 niemals stattgefunden hätte? Ein Gedankenexperiment. In: Zeit online, 11.08.2006. Quelle: https://www.zeit.de/online/2006/33/juli-zeh-9-11/komplettansicht (Zugriff: 27.07.2021).

1090 Borgans / Meißner / Zeh: „Ich bin ein großer Fan der Freiheit“. 


\subsection{Juli Zeh: Corpus Delicti}

In ihrem vierten Roman Corpus Delicti. Ein Prozess entwirft Juli Zeh die Vision einer Gesellschaft „,in der Mitte des einundzwanzigsten Jahrhunderts“ $(C D, 12)^{1091}$, welche die Gesundheit zum absoluten Wert sowie zum Prinzip staatlicher Legimitation erhoben hat. ${ }^{1092}$ Um die Gesundheit der Bevölkerung zu erhalten und zu steigern, hat die diktatorische Regierung des fiktiven Zukunftsdeutschland, die den Namen „die METHODE“ trägt, ein umfassendes Überwachungsregime errichtet. Ernährung und sportliche Aktivität der Individuen unterliegen strengen Kontrollen; selbst das Abwasser von Privathaushalten wird auf chemische Auffälligkeiten untersucht. Toxische Genussmittel sind streng verboten: Zigaretten und Alkohol, aber auch Tee und Kaffee gelten als harte Drogen und können nur auf illegalem Wege bezogen werden. Selbst die Wahl der Liebespartner folgt immunologischen Gesichtspunkten: Sexualkontakte zwischen zwei Personen werden nur dann gestattet, wenn die Abwehrsysteme der beiden kompatibel sind, was von der sogenannten ,Zentralen Partnerschaftsvermittlung“ (CD, 19) sichergestellt wird. Widersetzlichkeiten gegenüber den Forderungen des totalen Gesundheitsimperativs werden streng geahndet.

Im Zentrum der Romanhandlung stehen die Biologin Mia Holl, ihr Bruder Moritz sowie Heinrich Kramer, ein einflussreicher Journalist und Verteidiger der METHODE. Während Mia zu Beginn des Romans eine überzeugte Anhängerin des herrschenden Systems ist, lehnt Moritz die Bevormundung und Kontrolle der Individuen durch die Regierung ab und verteidigt das Recht auf körperliche Selbstverfügung, Rausch und leidenschaftliche Liebe. Nachdem Moritz fälschlicherweise eines Mordes angeklagt und verhaftet wurde, nimmt er sich im Gefängnis das Leben. Der Tod ihres Bruders stürzt Mia in eine tiefe Depression, in deren Folge sie ihre gesundheitlichen Meldepflichten vernachlässigt. Mias wiederholte Verstöße gegen die Auflagen des Gesundheitsregimes führen schließlich dazu, dass ein Strafprozess gegen sie angestrengt wird. Im Laufe des Verfahrens stellt sich jedoch heraus, dass Moritz nur deshalb mit einem DNA-Test des Mor-

1091 Zitate aus Corpus Delicti werden nach folgender Ausgabe mit der Sigle „CD“ im Text gegeben: Juli Zeh: Corpus Delicti. München 2010.

1092 Bereits in den beiden klassischen dystopischen Romanen Brave New World von Aldous Huxley und 1984 von George Orwell bildet die Einflussnahme auf Körper und Gesundheit ein zentrales Element der staatlich-totalitären Kontrolle über die Individuen. Vgl. Simone Schroth: "Bedrohung verlangt Wachsamkeit": Health and Healthcare as Instruments of Control in Two Recent Dystopias. In: Gillian Pye / Sabina Strümper-Krobb (Hg.): Germanistik in Ireland 9. Special Issue: Imagining Alternatives: Utopias - Dystopias - Heterotopias. Konstanz 2014, S. 121133, hier S. 121. 
des überführt werden konnte, weil er als Kind durch eine Knochenmarkspende von Leukämie geheilt wurde, was eine Veränderung seines genetischen Codes zur Folge hatte. Bei den DNA-Spuren, die sich in der Leiche des Opfers fanden, handelt es sich in Wahrheit um die DNA von Moritz' Knochenmarkspender. Aufgrund des posthumen Nachweises von Moritz' Unschuld gerät die METHODE, deren Legitimität gerade auf der Annahme ihrer „Unfehlbarkeit“ (CD, 37) beruht, unter Rechtfertigungsdruck. (Der Verweis auf die ,Unfehlbarkeit‘, wie sie realweltlich allenfalls dem Papst zugesprochen wird, bildet nur eine der zahlreichen Verbindungen von Gesundheitsideologie und religiösem Denken im Roman. ${ }^{1093}$ ) Daraufhin soll an Mia, die sich infolge des aufgedeckten Justizirrtums von der METHODE abwendet, ein Exempel statuiert werden: Der methodenfeindlichen Umtriebe und der Unterstützung einer terroristischen Vereinigung beschuldigt, wird Mia öffentlich diffamiert, gefoltert und schließlich zum Einfrieren auf unbestimmte Zeit verurteilt. Kurz vor der Vollstreckung des Urteils jedoch erfolgt überraschend die Begnadigung: Statt als Märtyrerin des Anti-Methodismus in die Geschichte einzugehen, wird Mia zur „Unterbringung in einer Resozialisierungsanstalt“, zur „Methodenlehre“ (CD, 264) - also zur ideologischen Indoktrination - und zur Wiedereingliederung in die totalitäre Gesellschaft verurteilt.

Die fiktionale Welt von Corpus Delicti bewegt sich in großer Nähe zur realen Welt der Leser: Die technischen Entwicklungen von Zehs Dystopie gehen über diejenigen der Gegenwart nur unwesentlich hinaus; auch wird der Übergang von der Gegenwart der Leser zur fiktiven Zukunft nicht eigens thematisiert. Auf eine funkelnde Science-Fiction-Staffage, wie sie für dystopische Texte durchaus charakteristisch ist, verzichtet der Roman weitgehend. ${ }^{1094}$ Insgesamt handelt es sich bei Corpus Delicti weniger um eine plausible „Linienverlängerung“

1093 Vgl. Carla Gottwein: Die verordnete Kollektividentität. Juli Zehs Vision einer Gesundheitsdiktatur im Roman Corpus Delicti. In: Corinna Schicht (Hg.): Identität. Fragen zu Selbstbildern, körperlichen Dispositionen und gesellschaftlichen Überformungen in Literatur und Film. 2., überarbeitete Aufl. Oberhausen 2012, S. 230-250, hier S. 236; Virginia McCalmont / Waltraud Maierhofer: Juli Zeh's Corpus Delicti (2009): Health Care, Terrorists, and the Return of the Political Message. In: Monatshefte 104/3 (2012), S. 375-392, hier S. 389.

1094 Auch Carrie Smith-Prei konstatiert: „While the novel does contain elements of science fiction and is set in the near future, the scientific advancements presented in the text are very much also those of today. “ (Carrie Smith-Prei: Relevant Utopian Realism: The Critical Corporeality of Juli Zeh’s Corpus Delicti. In: Peter M. McIsaac (Hg.): Visions of tomorrow. Science and utopia in German culture. Toronto, Ontario 2012, S. 107-123, hier S. 111) Vgl. auch Sabine Schönfellner: Die Perfektionierbarkeit des Menschen? Posthumanistische Entwürfe in Romanen von Juli Zeh, Kaspar Colling Nielsen und Margaret Atwood. Berlin 2018, S. 69; Layh: Finstere neue Welten, S. 158. 
(Adorno) ${ }^{1095}$ aktueller gesellschaftlicher Tendenzen in die Zukunft, sondern vielmehr, wie Marcus Schotte und Maja Vorbeck-Heyn schreiben, um eine ,verdeckte literarische Gegenwartsdiagnose in utopischem Gewand [...], die zur Auseinandersetzung mit zeitpolitischen Diskursen auffordert.“1096

Im Interview nach der realen Wahrscheinlichkeit ihrer dystopischen Szenarien befragt, antwortet Zeh:

Es ist aber tatsächlich so, dass ich das, was Sie mit ,Dystopie' meinen, also diese Corpus Delicti-Welt, überhaupt nicht als Dystopie empfinde. Also, das ist zwar in der Zukunft angesiedelt und lehnt sich deswegen ein bisschen in diese Science Fiction-Gattung hinein, aber ich habe dort eigentlich nichts erfunden, sondern ich habe nur Dinge, Tendenzen, Entwicklungen, die wir heute schon erleben, auf einen Haufen gekehrt [...]. Es ist nicht wirklich in die Zukunft gedacht, sondern es ist eine Gegenwartsverdichtung. Und deswegen ist es auch kein Pessimismus, sondern es ist einfach nur Diagnose [...]. Ich weiß nicht, was die Zukunft bringt, ich will es auch nicht wissen. Und ich glaube auch nicht, dass wir auf eine Gesundheitsdiktatur zusteuern - aber ich glaube, dass wir jetzt schon in Teilen eine sind. ${ }^{1097}$

Zeh stellt hier den Gegenwartsbezug, wie er für das Genre der Dystopie konstitutiv ist, in großer Deutlichkeit heraus: Nicht um eine plausible futurologische Entwicklungsdiagnose sei es ihr mit Corpus Delicti gegangen, sondern um eine

1095 Theodor W. Adorno: Aldous Huxley und die Utopie. In: Dialektik des Engagements. Frankfurt a. M. 1973, S. 151-178, hier S. 154.

1096 Marcus Schotte / Maja Vorbeck-Heyn: Die Zukunft unserer Gesellschaft liegt in ihrer Vergangenheit. Zu Juli Zehs Roman Corpus Delicti. Ein Prozess. In: Literatur im Unterricht. Texte der Gegenwartsliteratur für die Schule 12/2 (2011), S. 111-131, hier S. 129. Auch Gerigk weist die Zuordnung von Corpus Delicti zum Genre der Science-Fiction zurück: Der Akzent des Romans liege nicht auf der „avancierte[n] Technik einer Gesundheitsüberwachung, sondern [auf] den daraus resultierende[n] Wertkonflikte[n]“ (Anja Gerigk: „Die ideale Geliebte“ - Utopische Erzählformen im Spiegel neuer Dystopien (Zeh, Dath, Kracht). In: Alman Dili ve Edebiyatı Dergisi. Studien zur deutschen Sprache und Literatur 34/2 (2015), S. 5-16, hier S. 9, Anm. 4).

1097 Sebastian Horn: Fragen an Juli Zeh. In: Zeit online, 12.11.2011. Quelle: https://www.zeit.de/ video/2010-11/672198124001/videointerview-fragen-an-juli-zeh (Zugriff: 27.07.2021). Die prinzipielle Unmöglichkeit, Zukunftsentwicklungen verlässlich vorherzusagen, thematisiert Zeh in ihrer Rede Das Mögliche und die Möglichkeiten (2010): „Ich bin keine Untergangsprophetin; ich bin noch nicht einmal ein veritabler Pessimist. Der schwarze Abgrund ist keine Tatsache, sondern ein Bild, das der Zeitgeist malt, wenn er in die sogenannte Zukunft blickt. Für menschliche Wesen, deren Erkenntnisfähigkeit sich notwendig nur auf Vergangenes und Gegenwärtiges bezieht (und auch das nur in sehr eingeschränktem Maße), stellt die Zukunft immer eine Fiktion dar. Wir können nicht wissen, was kommt, wir können es uns nur vorstellen. Die Zukunft ist also per se Ansichtssache. Das gilt erst recht für ihre vorweggenommene Bewertung. “ (Zeh: Nachts sind das Tiere, S. 146). 
„Gegenwartsverdichtung“1098 respektive um eine, wie Sabine Schönfellner schreibt, „Gegenwartsdiagnose durch scheinbare Zukünftigkeit““ ${ }^{1099}$ Entsprechend konsequent wirkt es, dass Zeh die Nominierung ihres Romans für den Kurd-Laßwitz-Preis, den bedeutendsten Preis für Science-Fiction im deutschsprachigen Raum, abgelehnt hat. ${ }^{1100}$

Corpus Delicti ist in der Forschung umfassend kommentiert worden. ${ }^{1101}$ Die philosophischen und literarischen Bezüge des Textes ${ }^{1102}$, seine Behandlung der Körper-Thematik ${ }^{1103}$, seine juristische Dimension ${ }^{1104}$ sowie seine Mehrfachmedialisierung als Theaterstück, Roman und Hörnovelle ${ }^{1105}$ müssen

1098 Zur Gegenwartsanbindung von Zehs dystopischem Schreiben siehe auch Navratil: Jenseits des politischen Realismus, S. $370 \mathrm{f}$.

1099 Sabine Schönfellner: Erzählerische Distanzierung und scheinbare Zukünftigkeit. Die Auseinandersetzung mit biomedizinischer Normierung in Juli Zehs Romanen „Corpus Delicti“ und „Leere Herzen“. In: Zeitschrift für Germanistik NF 28/3 (2018), S. 540-554, hier S. 541.

1100 Vgl. Rolf Löchel: Die Zukunft ist weiblich. Quelle: https://literaturkritik.de/id/22558 (Zugriff: 27.07.2021). Im Interview hat Zeh auf die nationalspezifischen Erwartungen in Bezug auf die Gattung Science-Fiction hingewiesen: „Du kannst in Deutschland keinen SciencefictionRoman schreiben, der ,ernst“ ist. So etwas wie die Texte Stanislaw Lems wäre undenkbar in Deutschland.“ (Zeh: Der Unterschied zwischen Realität und Fiktion ist marginal, S. 38f.).

1101 Im Januar 2020 listet die Bibliografie der deutschen Sprach- und Literaturwissenschaft (BDSL) 26 Einträge zu Zehs Corpus Delicti, mehr als zu jedem anderen Werk der Autorin.

1102 Vgl. Gottwein: Die verordnete Kollektividentität; Achim Geisenhanslüke: Die verlorene Ehre der Mia Holl. Juli Zehs Corpus Delicti. In: Viviana Chilese / Heinz-Peter Preusser (Hg.): Technik in Dystopien. Heidelberg 2013, S. 223-232; Stefan Neuhaus: Konsequenzen der Biopolitik: Zum Verhältnis von Subjekt und Umwelt in literarischen Dystopien. In: Martin Hellström / Linda Karlsson Hammarfelt / Edgar Platen (Hg.): Umwelt - sozial, kulturell, ökologisch. Zur Darstellung von Zeitgeschichte in deutschsprachiger Gegenwartsliteratur (IX). München 2016, S. 109-130.

1103 Vgl. Sarah Koellner: Data, Love, and Bodies: The Value of Privacy in Juli Zeh's Corpus Delicti. In: Seminar. A Journal of Germanic Studies 52/4 (2016), S. 407-425; Smith-Prei: Relevant Utopian Realism.

1104 Vgl. Heinz Müller-Dietz: Strafrecht im Zukunftsstaat? Zur negativen Utopie in Juli Zehs Roman „Corpus Delicti“. In: Claudius Geisler u. a. (Hg.): Festschrift für Klaus Geppert zum 70. Geburtstag am 10. März 2011. Berlin / New York 2011, S. 423-439; Thomas Weitin: Ermittlung der Gegenwart: Theorie und Praxis unsouveränen Erzählens bei Juli Zeh. In: Postsouveränes Erzählen. Zeitschrift für Literaturwissenschaft und Linguistik 165/42 (2012), S. 67-86.

1105 Vgl. Christopher Schmidt: Die Erfindung der Realität. Über Juli Zehs Erstlingsstück Corpus delicti. In: Sprache im technischen Zeitalter 46/187 (2008), S. 263-269; Birte Giesler: „Das Mittelalter ist keine Epoche. ,Mittelalter“ ist der Name der menschlichen Natur.“ Zeitgenössisches Drama als rückwärts gekehrte Dystopie in Juli Zehs Corpus Delicti. In: Wolfgang Braungart / Lothar van Laak (Hg.): Gegenwart Literatur Geschichte. Zur Literatur nach 1945. Heidelberg 2013, S. 265-293; Elisabeth Tropper: Analytische Apokalyptiker. Überlegungen zum Dystopischen in Theatertexten von Falk Richter und Juli Zeh. In: Gillian Pye / Sabina Strüm- 
an dieser Stelle kein weiteres Mal erläutert werden. Stattdessen soll eine Interpretation vorgelegt werden, welche die Realitätsbezüge des Romans in den Blick nimmt und dabei insbesondere die Frage zu beantworten sucht, welchen Beitrag die kontrafaktischen Realitätsvariationen zum politischen Projekt von Corpus Delicti leisten.

Im Folgenden wird zunächst Zehs Auseinandersetzung mit den Themen Gesundheitswahn, Datenüberwachung und Bürgerrechten in faktualen Texten diskutiert: in Interviews, Zeitungsartikeln und einem Sachbuch. Zehs Einlassungen zu Themen des öffentlichen, politischen Diskurses können insofern als ,Arbeit am Kontext' begriffen werden, als die Autorin hier realweltliches Faktenmaterial aufbereitet und in den Fokus der Öffentlichkeit rückt, Faktenmaterial, welches sie dann wiederum in ihrem literarischen Werk (variierend) aufgreift. Anschließend wird die ideologische Ordnung der fiktionalen Welt von Corpus Delicti anhand der miteinander korrespondierenden Themenkomplexe Biopolitik und Normalismus in den Blick genommen. Die Auseinandersetzung mit der Frage, was als normal und was als abseitig gilt, leitet über zu einer Erläuterung der (kontrafaktischen) Mittelalterbezüge im Roman. Insbesondere über das Thema der Folter werden hier signifikante Bezüge zwischen der futurischen Diegese des Romans und der Gegenwart seines Erscheinens eröffnet. Abschließend soll die politische ,Botschaft‘ von Corpus Delicti unter besonderer Berücksichtigung ihrer literarischen Inszenierungsweise diskutiert und die These von der Eindeutigkeit dieser Botschaft einer kritischen Prüfung unterzogen werden.

\subsubsection{Sekundierende Faktenerzeugung: Juli Zehs Arbeit am Kontext}

Wie bei allen kontrafaktischen Texten muss auch bei kontrafaktischen Dystopien die Frage gestellt werden, auf welches realweltliche Faktenmaterial hier genau Bezug genommen wird. ${ }^{1106}$ Im Falle von Zehs Corpus Delicti ist die Frage nach den alludierten Fakten insofern von gesteigertem Interesse, als Zeh sich

per-Krobb (Hg.): Germanistik in Ireland 9. Special Issue: Imagining Alternatives: Utopias Dystopias - Heterotopias. Konstanz 2014, S. 135-150.

1106 Die Überlegungen dieses Kapitels habe ich ausführlicher entwickelt in Michael Navratil: Die doppelte Autorität der Autoren zwischen Fiktionalität und Faktualität. Die Causa Robert Menasse und Juli Zehs Dystopien. In: Vera Podskalsky / Deborah Wolf (Hg.): Prekäre Fakten, umstrittene Fiktionen. Fake News, Verschwörungstheorien und ihre kulturelle Aushandlung. Philologie im Netz Beiheft 25/2021, S. 163-188, URL: http://web.fu-berlin.de/phin/beiheft25/ b25t07.pdf (Zugriff: 27.07.2021). 
bei den Themen Gesundheitsdiktatur, Überwachungsstaat und Bürgerrechte nicht darauf beschränkt hat, mehr oder weniger allgemein bekannte gesellschaftliche Tendenzen in einem fiktionalen Medium aufzugreifen; sie hat darüber hinaus auch in einer Reihe faktualer Texte - Interviews, Zeitungsartikeln und einem Sachbuch - das öffentliche Interesse auf ebendiese gesellschaftlichen Tendenzen zu lenken versucht. In unterschiedlichen Textsorten und Äußerungskontexten kritisiert Zeh die Entwicklungstendenz einer Gesellschaft, welche die Erhaltung und Steigerung der Gesundheit in zunehmendem Maße zur individuellen Pflicht erhebt, in der Menschen den eigenen Körper immer stärker zu kontrollieren suchen - etwa durch Fitness oder die Vermeidung von Genussmitteln - und in der Gesundheitsvorstellungen zusehends objektiviert und statistisch normiert werden. Als gefährlich schätzt Zeh diese Entwicklungen ein, weil durch einen standardisierten Gesundheitsbegriff die Entscheidungsfreiheit darüber, was der Einzelne als gesund empfindet und wie er die Gesundheit als Wert ins Verhältnis zu anderen Werten setzen will, unterminiert zu werden droht. ${ }^{1107}$ Indem die Gesundheit, so Zeh, immer fragloser als „das höchste Gut" ${ }^{\prime 1108}$ angesehen wird, geraten andere gesellschaftliche Prioritäten aus dem Blick, etwa „Hochleistung in der Kultur“"1109, aber auch „Liebe, Solidarität vielleicht, Verantwortung für andere“ “. ${ }^{1110}$ In den Plänen etwa, Krankenkassenbeiträge an das individuelle Gesundheitsprofil anzupassen - also einerseits Ermäßigungen für Personen einzuführen, die ihre Fitnessaktivitäten durch Apps überwachen lassen, und andererseits höhere Beiträge von Personen zu verlangen, die einen vermeintlich ungesunden Lebensstil pflegen -, erkennt Zeh eine Tendenz, „Krankheit mit Schuld [zu] identifizieren“, sodass, „wer sich selbstverschuldet verletzt, keinen Anspruch mehr auf Fürsorge hat."“1111

Zeh formuliert ihre Kritik am Gesundheits- und Fitnesswahn zum einen in kleinen Textgattungen wie Interview und Zeitungsartikel. Zum anderen hat Zeh im Jahre 2009 - also im Jahr des Erscheinens von Corpus Delicti - zusammen

1107 So hält Schroth fest: „[I]t could be said that any system declaring one single value as central is in danger of becoming dystopian, meaning that its negative, limiting elements outweigh what is gained by adopting the rules prescribed by the system. This dilemma, especially in the case of Juli Zeh, is voiced not only by the protagonist [of Corpus Delicti] but also when the authors express their opinion outside their works of fiction." (Schroth: "Bedrohung verlangt Wachsamkeit”, S. 124).

1108 Kathrin Hondl / Juli Zeh: Ein Plädoyer gegen den Gesundheits- und Fitnesswahn. 08.01.2012. Quelle: https://www.deutschlandfunk.de/ein-plaedoyer-gegen-den-gesund heits-und-fitnesswahn.691.de.html?dram:article_id=56526 (Zugriff: 27.07.2021).

1109 Gernert / Zeh: Plädoyer gegen die Fitness-Diktatur.

1110 Hondl / Zeh: Ein Plädoyer gegen den Gesundheits- und Fitnesswahn.

1111 Gernert / Zeh: Plädoyer gegen die Fitness-Diktatur. 
mit dem Schriftstellerkollegen Ilija Trojanow ein Sachbuch mit dem Titel Angriff auf die Freiheit. Sicherheitswahn, Überwachungsstaat und der Abbau bürgerlicher Rechte veröffentlicht. Von den Autoren als problematisch eingeschätzte Entwicklungen im Bereich des Gesundheitswesens sind nur eines der Themen, das diese politische Streitschrift verhandelt. Allgemein warnen Trojanow und Zeh vor den Folgen eines „Wettrüsten[s] beim Thema Sicherheit“"1112, einer immer weitergehenden Durchleuchtung der Bürger und dem Schwinden individueller Freiheiten. Dem Abbau bürgerlicher Rechte begegnen die Autoren mit einem klaren politischen Appell:

Ein Frosch, der in einen Topf mit heißem Wasser geworfen wird, springt sofort wieder heraus, wenn er kann. Doch setzt man ihn in kaltes Wasser und erwärmt den Topf gleichmäßig, bleibt er ruhig sitzen, bis er stirbt.

Wir haben in unserer Geschichte genügend Frösche als warnende Beispiele vor Augen. Wenn wir uns jetzt nicht wehren, werden wir späteren Generationen nur schwer erklären können, warum wir nicht in der Lage waren, ihnen eine Freiheit zu vererben, die wir einst selbst genossen. Seit 2001 schauen wir wie gelähmt zu, was in und mit unserem Land passiert, während man uns einzureden versucht, die Lehren des 20. Jahrhunderts hätten im 21. Jahrhundert nichts mehr zu bedeuten. Raus aus dem Topf! $!^{1113}$

Die Autoren fordern die Leser ihres Buches dazu auf, eine „Sensibilität für den Wert [von] Daten und [...] Intimsphäre“ zu entwickeln, mahnen einen kritischen Umgang mit Politikeraussagen an, sobald darin „Begriffe wie ,Terrorismus“ und ,Terrorverdächtiger“ fallen“, und verteidigen generell die Freiheitsrechte des Einzelnen. ${ }^{1114}$ Diese Verteidigung individueller Freiheiten begründen Trojanow und Zeh mit einem ,unbestreitbare[n] Erfahrungswert: Die totalitären Systeme des 20. Jahrhunderts haben [...] alle konservativen Kontrollphantasien seit Hobbes diskreditiert. Denn die Auswirkungen staatlicher Übermacht haben sich als unendlich viel schlimmer erwiesen als jede individuelle Verfehlung.“"1115

Insgesamt lassen Zehs Interviews, Zeitungsartikel, das zusammen mit Ilija Trojanow verfasste Sachbuch sowie die literarischen Dystopien der Autorin eine kohärente politische Agenda erkennen. Zehs faktuale Texte weisen dabei

1112 Ilija Trojanow / Juli Zeh: Angriff auf die Freiheit. Sicherheitswahn, Überwachungsstaat und der Abbau bürgerlicher Rechte. München 2009, S. 134.

1113 Trojanow / Zeh: Angriff auf die Freiheit, S. 16f.

1114 Trojanow / Zeh: Angriff auf die Freiheit, S. 138.

1115 Trojanow / Zeh: Angriff auf die Freiheit, S. 27. Derselbe Gedanke findet sich auch in Zehs Theaterstück Der Kaktus: „[D]ie größten Verbrechen [werden] immer noch von Staaten begangen [...]. Man muss den Bürger vor dem Staat schützen, nicht den Staat vor dem Bürger.“ (Juli Zeh: Good Morning, Boys and Girls. Theaterstücke. Frankfurt a. M. 2013, S. 46). 
naturgemäß ein höheres Maß an argumentativer Stringenz, eine größere Explizitheit der politischen Botschaft sowie Belege aus der Forschung auf; Zehs fiktionale Texte hingegen operieren eher mit Zuspitzungen und Andeutungen und erheben qua Fiktionalität naturgemäß keinen Anspruch auf direkte realweltliche Referentialisierbarkeit. Der politische Anspruch jedoch, nämlich der Versuch einer Verteidigung individueller Freiheitsrechte, ist über die unterschiedlichen Gattungen und Medien hinweg konstant.

Die parallele Behandlung derselben Themen in faktualen und fiktionalen Äußerungskontexten ist im Zusammenhang mit der Kontrafaktik von besonderem Interesse, beruht das Erzählphänomen doch gerade auf einer Vermittlung faktualer und fiktionaler Geltungsansprüche. Zeh greift auf faktuale Medien wie Zeitungsartikel oder Sachbuch zurück, um auf realweltliche Fakten hinzuweisen, ja ,erzeugt' solche Fakten in gewissem Sinne sogar, insofern sie bestimmte Sachverhalte durch ihre lautstarke Kritik in den Aufmerksamkeitskreis eines größeren Teils der Öffentlichkeit rückt. Der Anspruch auf intersubjektive Überprüfbarkeit bleibt dabei in Zehs faktualen Texten jedoch stets gewahrt: ${ }^{1116}$ Die Autorin spricht hier nie als ,Dichterprophetin“, sondern stets als engagierte Bürgerin. ${ }^{1117}$

In einem zweiten Schritt können diejenigen Fakten, die Zeh in ihren faktualen Texten präsentiert, dann allerdings durchaus in fiktionalen Formaten aufgegriffen und dort mitunter auch variiert werden (wobei Täuschungsabsichten zuverlässig ausgeschlossen werden können, wurde eine Kenntnis der Fakten doch gerade durch deren korrekte Präsentation in faktualen Formaten sichergestellt). Zeh nutzt somit ihre privilegierte Sprecherposition als erfolgreiche Autorin, um im öffentlichen Diskurs bestimmten gesellschaftlichen oder politischen Entwicklungen zu besonderer Aufmerksamkeit zu verhelfen. (Mit Pierre Bourdieu könnte man hier von einem Einsatz der im literarischen Feld erworbe-

1116 In ihrer Tübinger Poetikvorlesung bemerkt Zeh hierzu: „Wer gegen einen herrschenden Diskurs anschreiben will, muss sich der gleichen Mittel bedienen wie die Gegenseite, wenn er gehört werden will - wobei die Grenze des Erlaubten durch Faktengenauigkeit markiert wird.“ (Juli Zeh / Georg M. Oswald: Aufgedrängte Bereicherung. Tübinger Poetik-Dozentur 2010. Künzelsau 2011, S. 85).

1117 Wagner fasst Zehs Äußerungen zu ihrer eigenen, öffentlichen und politischen Positionierung wie folgt zusammen: „Ihre Bekanntheit als Schriftstellerin verschaffe ihr, und das markiert sie als (den einzigen) Unterschied zwischen sich und den anderen Bürgern, die notwendige Aufmerksamkeit, in der Öffentlichkeit gehört zu werden. Aber sie spricht, so soll man ihre Eingaben verstehen, mit diesem Privileg immer als eine von vielen“ (Wagner: Aufklärer der Gegenwart, S. 64). Siehe zu Zehs poetologischem Selbstentwurf als Schriftstellerin und Bürgerin ausführlich ebd., S. 101-106. 
nen Autorität im politischen Feld sprechen. ${ }^{1118}$ ) Diese Entwicklungen geben dann wiederum das Faktenmaterial für Zehs fiktionale Texte ab. ${ }^{1119}$ Vom literarischen Werk aus betrachtet lässt sich Zehs außerliterarisches Engagement somit als ,Arbeit am Kontext‘ begreifen, also als sekundierende Faktenerzeugung und Aufbereitung von Informationen, auf die dann in den Romanen und Theaterstücken Bezug genommen werden kann: sei es in Form einfacher Anspielungen, sei es in Form kontrafaktischer Variationen.

\subsubsection{Biopolitik und Normalismus}

Als Vorwort ist dem Roman Corpus Delicti ein längeres Zitat aus dem fiktiven Werk Gesundheit als Prinzip staatlicher Legitimation vorangestellt, welches mit dem folgenden Satz beginnt: „Gesundheit ist ein Zustand des vollkommenen körperlichen, geistigen und sozialen Wohlbefindens - und nicht die bloße Abwesenheit von Krankheit“ (CD, 7). Bereits dieser erste Satz stellt eine Verbindung her zwischen der futurisch-fiktionalen Welt des Romans und der Gegenwart der Leser, handelt es sich hier doch um ein fast wörtliches Zitat aus der Verfassung der Weltgesundheitsorganisation. In deren zweitem Abschnitt heißt es: „Die Gesundheit ist ein Zustand des vollständigen körperlichen, geistigen und sozialen Wohlergehens und nicht nur das Fehlen von Krankheit oder Gebrechen. " ${ }^{1120}$ In ihrem weiteren Verlauf bezeichnet die WHO-Verfassung dann den „Besitz des bestmöglichen Gesundheitszustandes“ als „eines der Grundrechte jedes menschlichen Wesens““. ${ }^{1121}$ Gesundheit wird hier also als ein natürliches Recht betrachtet, sie wird an den einzelnen Menschen gebunden (,eines der Grundrechte jedes menschlichen Wesens“) und in ihrer tendenziellen Unverfügbarkeit (,,bestmögliche[r] Gesundheitszustand[...]“) anerkannt.

In der fiktionalen Welt von Corpus Delicti hingegen sind diese Rechtsprinzipien ausgesetzt: Gesundheit wird im Roman wesentlich mit Blick auf die Gesellschaft

1118 Vgl. Pierre Bourdieu: Die Regeln der Kunst. Genese und Struktur des literarischen Feldes. Frankfurt a. M. 1999, S. 210 f.

1119 So hält Koellner fest: ,[W]hen Corpus Delicti is read together with Angriff auf die Freiheit and Nachts sind das Tiere, both nonfictional works become a commentary and metatext of Corpus Delicti, which open up a politically engaged reading of Zeh's work and plays an important role in the reader's assessment of Mia Holl's case“ (Koellner: Data, Love, and Bodies, S. 412).

1120 Die ursprüngliche Verfassung der WHO wurde am 22. Juli 1946 in New York unterzeichnet. Für die aktuelle Fassung (08.05.2014) siehe: Verfassung der Weltgesundheitsorganisation. Quelle: https://www.admin.ch/opc/de/classified-compilation/19460131/201405080000/ 0.810.1.pdf (Zugriff: 27.07.2021).

1121 Verfassung der Weltgesundheitsorganisation. 
konzeptualisiert (wobei die ,Gesellschaft' hier eher in Form einer völkisch-entindividualisierten ,Gemeinschaft‘ erscheint), sie wird als instrumentell herstellbar begriffen und ihre Steigerung wird zur individuellen sowie kollektiven Pflicht erklärt:

Gesundheit ist nicht Durchschnitt, sondern gesteigerte Norm und individuelle Höchstleistung. Sie ist sichtbar gewordener Wille, ein Ausdruck von Willensstärke in Dauerhaftigkeit. Gesundheit führt über die Vollendung des Einzelnen zur Vollkommenheit des gesellschaftlichen Zusammenseins. Gesundheit ist das Ziel des natürlichen Lebenswillens und deshalb natürliches Ziel von Gesellschaft, Recht und Politik. Ein Mensch, der nicht nach Gesundheit strebt, wird nicht krank, sondern ist es schon.

(CD, $7 \mathrm{f}$.)

Gesundheit fungiert hier als zentrales Prinzip der gesellschaftlichen Organisation. ,Gesundheit' und ,Krankheit' bezeichnen dabei offenkundig mehr als nur körperliche Zustände: Sie sind ideologisch massiv überkodiert. In der Gesundheitsdiktatur von Corpus Delicti erscheint bereits das Fehlen einer Gesinnung, welche mit den Grundsätzen der METHODE übereinstimmt, als krankhaft. So behauptet Heinrich Kramer im Rahmen seiner Hetzkampagne gegen Mia Holl: „Heutzutage [...] bestünden die gefährlichsten Viren nicht mehr aus Nukleinsäuren, sondern aus infektiösen Gedanken.“ (CD, 200)

Rein thematisch lassen sich in Corpus Delicti zwei politische Komplexe voneinander unterscheiden, welche in anderen Texten der Autorin mitunter auch getrennt voneinander behandelt werden ${ }^{1122}$ : erstens die Überbetonung der Gesundheit und zweitens die Einschränkung bürgerlicher Rechte durch flächendeckende Überwachung, Datensammlung oder gar strategisch lancierte Terrorbeschuldigungen und Folter. Das kritische Potenzial des Romans erwächst wesentlich aus den vielfältigen Verschränkungen dieser beiden Komplexe: ${ }^{123}$ Die Zielsetzung der gesamtgesellschaftlichen Gesundheitssteigerung macht eine flächendeckende Überwachung notwendig; der Umstand, dass die Gesundheit als oberster, natürlicher Wert

1122 Zehs Stück Der Kaktus etwa verhandelt das Thema der Folter von Terrorverdächtigen. Das Thema Gesundheit spielt dort hingegen keine Rolle.

1123 Es lässt sich hierin - wie generell über weite Strecken des Romans - ein Anschluss an Giorgio Agambens Versuch erkennen, den „verborgenen Kreuzpunkt zwischen dem juridischinstitutionellen Modell und dem biopolitischen Modell der Macht“ in modernen Gesellschaften herauszuarbeiten (Giorgio Agamben: Homo sacer. Die Souveränität der Macht und das nackte Leben, Frankfurt a. M. 2002, S. 16). Christopher Schmidt zufolge kann „die Auseinandersetzung mit der Deutungshoheit des italienischen Philosophen Giorgio Agamben [...] wohl als Urzelle von Corpus delicti betrachtet werden.“ (Schmidt: Die Erfindung der Realität, S. 266) Die unterschiedlichen Vorschläge der Forschung zu möglichen Verbindungen von Zehs Roman mit Agambens Theorien sammelt Schönfellner: Erzählerische Distanzierung und scheinbare Zukünftigkeit, S. 543f. 
der Gesellschaft angesehen wird, führt dazu, dass diejenigen Personen, die diesem Wert nicht huldigen, aus der Bürgergemeinschaft ausgeschlossen werden und in der Folge öffentlicher Diffamierung, politischer Verfolgung oder gar Folter ausgesetzt sind; und die Daten, die vorgeblich zum Zweck der Gesundheitsvorsorge des Einzelnen und der Gemeinschaft erhoben werden, ermöglichen zugleich die Bespitzelung politisch Andersdenkender. Kritisiert wird somit alles in allem nicht so sehr der Wert der Gesundheit an sich, sondern die Absolutsetzung dieses Wertes, welche eine Einschränkung individueller Freiheitsrechte, im Extremfall gar die Errichtung eines totalitären Staatssystems zu legitimieren droht. ${ }^{1124}$ Mit einem Begriff von Petr Skrabanek könnte man hier von einer Diktatur unter dem Vorzeichen des „healthism“ sprechen. ${ }^{1125}$ Der totalitäre Gesundheitsimperativ wird in der fiktionalen Welt von Corpus Delicti zur Grundlage der Diktatur.

Der Absolutheitsanspruch der staatlichen Ideologie deutet sich bereits im Begriff ,METHODE' an: Ein politischer oder weltanschaulicher Inhalt, wie er in der Regel Teil des Namens politischer Parteien ist, muss hier gar nicht mehr angezeigt werden, da Alternativen zur dominanten Regierungsform ohnehin nicht zugelassen sind. Statt eines politischen Programms wird nur noch die ,Methode“ benannt, welche zur Durchsetzung der Interessen des Staates eingesetzt wird (bezeichnenderweise bleibt unklar, ob mit dem Begriff ,METHODE` ein Staatssystem, die Regierung selbst, ein bestimmtes Vorgehen dieser Regierung oder ein Rechtsgrundsatz gemeint ist ${ }^{1126}$ ). Die Legitimität dieses Staates gründet dabei wesentlich auf dem Anspruch, mit den eigenen Zielsetzungen direkt den Forderungen der Vernunft zu entsprechen. So proklamiert Kramer: „Wir gehorchen allein der Vernunft, indem wir uns auf eine Tatsache berufen, die sich unmittelbar aus der Existenz von biologischem Leben ergibt. Denn ein Merkmal ist jedem lebenden Wesen zu

1124 In einem Essay aus dem Jahr 2005 betont Zeh: „Ob Anti-Terror-Kampf vs. Datenschutz, Softwarepatente vs. open source, physische Selbstbestimmung vs. Gesundheitspolitik oder Sterberecht vs. Euthanasieverbot - hinter vielen politischen Diskussionen der Gegenwart verbirgt sich der Widerstreit zwischen dem Konzept individueller Freiheit auf der einen und jenem von staatlich herbeigeführter Sicherheit und Kontrollierbarkeit auf der anderen Seite. Diese beiden Werte ergänzen und begrenzen sich; bis zu einem gewissen Grad schließen sie sich sogar gegenseitig aus.“ (Juli Zeh: Alles auf dem Rasen. Kein Roman. Frankfurt a. M. 2008, S. 24).

1125 Vgl. Petr Skrabanek: The Death of Human Medicine and the Rise of Coercive Healthism. Bury St Edmunds 1994.

1126 Der unklare Status der METHodE lässt sich im Roman etwa an der Variation bekannter Formulierungen aus dem juristischen oder politischen Bereich erkennen: So ergehen Urteile in der fiktionalen Welt „IM NAMEN DER Methode!“ (CD, 9), es gibt aber auch einen „Methodenschutz“ (CD, 73) und (vermeintliche) „Methodenfeinde“ (CD, 206). 
eigen. Es zeichnet jedes Tier und jede Pflanze und erst recht den Menschen aus: Der unbedingte, individuelle und kollektive Überlebenswille.“ (CD, 36) Die enge Kopplung von individuellen und kollektiven Interessen, wie Kramer sie hier behauptet und wie sie auch an anderen Stellen im Roman immer wieder hervorgehoben wird, schließt einen bloß individuellen Umgang mit Fragen von Krankheit und Gesundheit aus. So gerät Mia zunächst nicht etwa darum in Konflikt mit den Organen des Staates, weil sie auf eine Abschaffung des herrschenden Systems hinarbeiten würde, sondern allein deshalb, weil sie den Schmerz angesichts des Todes ihres Bruders als „eine Privatangelegenheit“ (CD, 54) betrachtet wissen will und entsprechend für sich in Anspruch nimmt, auf ganz individuelle Weise um Moritz trauern zu dürfen. Vom Gericht muss sich Mia dann allerdings belehren lassen:

Es liegt in Ihrem Interesse, jede Form von Krankheit zu vermeiden. In diesem Punkt decken sich Ihre Interessen mit jenen der MEтHоDE, und auf diese Übereinstimmung stützt sich unser gesamtes System. Es besteht eine enge Verbindung zwischen dem persönlichen und dem allgemeinen Wohl, die in solchen Fällen keinen Raum für Privatangelegenheiten lässt.

$(\mathrm{CD}, 58)$

Nun bildet eine derartige enge Kopplung des staatlichen Interesses an das physische Wohl des Einzelnen ganz generell ein Charakteristikum moderner Gesellschaften, wie insbesondere Michel Foucault gezeigt hat. Seit der Mitte des 18. Jahrhunderts sieht Foucault eine Form der Macht sich formieren, „deren höchste Funktion nicht mehr das Töten, sondern die vollständige Durchsetzung des Lebens ist“"

Die Fortpflanzung, die Geburten- und die Sterblichkeitsraten, das Gesundheitsniveau, die Lebensdauer, die Langlebigkeit mit allen ihre Variationsbedingungen wurden zum Gegenstand eingreifender Maßnahmen und regulierender Kontrollen: Bio-Politik der Bevölkerung. Die Disziplinen des Körpers und die Regulierungen der Bevölkerung bilden die beiden Pole, um die herum sich die Macht zum Leben organisiert hat. ${ }^{1127}$

Genau eine solche Verbindung des einzelnen Körpers mit dem ,Gesamtkörper` der Gesellschaft - fast möchte man sagen: mit dem ,Volkskörper ${ }^{6}$ - wird in der fiktionalen Welt von Corpus Delicti konsequent zu Ende gedacht. Biopolitik scheint hier den gesamten Bereich des Politischen durchdrungen zu haben: Politik, insofern damit der Einfluss des Staates auf seine Bürger bezeichnet wird, ist in Zehs Roman wesentlich Biopolitik. Die kollektive Disziplinierung der Körper wird dabei derart ins Extrem getrieben, dass die entworfene Gesellschaft erkennbar totalitäre

1127 Michel Foucault: Der Wille zum Wissen. Sexualität und Wahrheit 1. Frankfurt a. M. 1983, S. 135. 
Züge annimmt: von der Abwehr des bloß Individuellen über die umfassende gesundheitliche Kontrolle der Bevölkerung bis hin zur gegenseitigen Bespitzelung der Bürger. Man geht wohl nicht zu weit, in dem Begriff „Santé“ (erstmals CD, 15), welcher die gängige Grußformel innerhalb der fiktionaler Welt bildet, eine kontrafaktische Variation des ,Heils'-Grußes faschistischer Regime zu erkennen: In der kontrafaktischen Variation dieses Grußes verbinden sich die ideologisch-messianische Dimension des ,Heils ‘ mit dessen biopolitischer Komponente ${ }^{1128}$, sodass der diktatorische Charakter des herrschenden Gesundheitsregimes deutlich hervortritt.

Zur Charakterisierung der fiktiven Gesellschaft in Corpus Delicti greift Zeh vielfach auf, wie Susanne Layh schreibt, ,in der zeitgenössischen Gegenwart

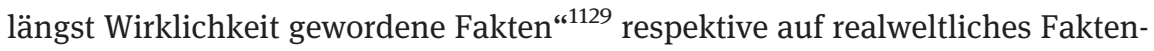
material zurück: so etwa auf Vorratsdatenspeicherung, die flächendeckende Überwachung durch die NSA, die Zurückdrängung des Solidaritätsgedankens im Krankenversicherungswesen sowie auf die Rauchverbote in öffentlichen Räumen. ${ }^{1130}$ In der fiktionalen Welt des Romans werden all diese Aspekte der Gegenwart auf zweifache Weise kritisch kommentiert: erstens, indem ihre negative Dimension durch Übertreibungen - respektive kontrafaktische Zuspitzung - besonders deutlich herausgestellt wird, und zweitens, indem die genannten Einzelentwicklungen durch eine funktionale Einbindung in ein totalitäres System, welches die Rechte des Individuums empfindlich einschränkt und mitunter sogar auf das Mittel der Folter zurückgreift, gleichsam metonymisch diskreditiert werden.

Besonders eindrücklich lässt sich die oppressive Wirkung der „Bio-Macht“1131 anhand des ambivalenten Komplexes Norm/Normalität/Normativität im Roman herausstellen. Für Kramer als erklärten Anhänger der METHODE bilden Gesundheit und Normalität weitgehend austauschbare Begriffe: „Was sollte vernünftigerweise dagegen sprechen, Gesundheit als Synonym für Normalität zu betrachten? Das Störungsfreie, Fehlerlose, Funktionierende: Nichts anderes taugt zum Ideal.“

$1128 \mathrm{Zu}$ den unterschiedlichen semantischen und normativen Dimensionen des Begriffs der Gesundheit siehe Letizia Dieckmann / Julian Menninger / Michael Navratil: Gesundheit und Erzählen: Zur Einleitung. In: Dies. (Hg.): Gesundheit erzählen. Ästhetik - Performanz - Ideologie. Berlin / Boston 2021 (in Vorbereitung).

1129 Layh: Finstere neue Welten, S. 159.

1130 Die Übereinstimmungen reichen dabei bis auf die Ebene sprachlicher Details. Nover zufolge etabliert Corpus Delicti eine „unmittelbare Beziehung zu den realen sicherheitspolitischen Diskussionen des Jahres 2007“, und zwar indem der Text konkrete Äußerungen des damaligen deutschen Innenministers Wolfgang Schäuble zum Thema Terrorismus sprachlich „variier[t]“. Vgl. Immanuel Nover: Der disziplinierte Körper. Ethik, Prävention und Terror in Juli Zehs Corpus Delicti. Ein Prozess. In: Kritische Ausgabe 17/24 (2013), S. 79-84, hier S. 81.

1131 Foucault: Der Wille zum Wissen, S. 135. 
(CD, 181) Mia hingegen erkennt im Laufe der Handlung, dass es sich bei dem Konzept der Normalität um ein ,zweischneidiges Schwert` handelt:

Die METHODE gründet sich auf die Gesundheit ihrer Bürger und betrachtet Gesundheit als Normalität. Aber was ist normal? Einerseits alles, was der Fall ist, das Gegebene, Alltägliche. Andererseits aber bedeutet „normal“ etwas Normatives, also das Gewünschte. Auf diese Weise wird Normalität zu einem zweischneidigen Schwert. Man kann den Menschen am Gegebenen messen und zu dem Ergebnis kommen, er sei normal, gesund und folglich gut. Oder man erhebt das Gewünschte zum Maßstab und stellt fest, dass der Betreffende gescheitert ist. Ganz nach Belieben. Solange man dazugehört, dient dieses Schwert der Verteidigung. Befindet man sich draußen, stellt es eine schreckliche Bedrohung dar. Es macht krank.

$(\mathrm{CD}, 145)$

Die normativ-deskriptive Doppelnatur des Normalitätsbegriffs lässt sich mit Jürgen Links Unterscheidung zwischen „Protonormalismus“ und „Flexibilitätsnormalismus“"1132 genauer fassen: Während der Protonormalismus eine „Tendenz zur ,Anlehnung‘ der Normalität an Normativität“ zeigt, zu „fixe[n] Normal- und Grenzwerte[n]“ tendiert und eine „,harte“ semantische und symbolische Markierung der Grenze“ zum Nicht-Normalen vornimmt, zeichnet sich der Flexibilitätsnormalismus durch „dynamische und in der Zeit variable Grenze[n]“, durch eine „,Entfernung“ der Normalität von [der] Normativität“ sowie durch ein „Floating“ von „flexible[n] und dynamische[n] Normal- und Grenzwerte“ aus. ${ }^{1133}$ Zwar schließen sich diese beiden Normalismus-Modelle keineswegs gegenseitig aus. Doch konstatiert Link für die zweite Hälfte des 20. Jahrhunderts eine deutliche Zunahme an flexibel-normalistischen Strukturen: ${ }^{1134}$ Anstatt von einer gegebenen Norm auszugehen, orientiert sich die Definition dessen, was als ,normal gilt, zusehends an einer normativ neutralen Beobachtung der Realität, mit all ihren Übergängen, weichen Grenzen und dynamischen ,Normal'verteilungen.

Angesichts einer solchen Dominanz flexibel-normalistischer Strukturen in der Gegenwart muss eine Rückkehr zu protonormalistischen Strukturen unausweichlich als Freiheitseinschränkung empfunden werden. So weist etwa HansGeorg Gadamer in seinem Essay Über die Verborgenheit der Gesundheit - einem der überraschend wenigen philosophischen Texte, die sich explizit mit Gesundheit (und nicht mit Krankheit ${ }^{1135}$ ) auseinandersetzen - auf die Gefahren des

1132 Jürgen Link: Versuch über den Normalismus. Wie Normalität produziert wird. 3., ergänzte, überarbeitete und neu gestaltete Aufl. Göttingen 2006, S. 51.

1133 Link: Versuch über den Normalismus, S. 57.

1134 Vgl. Link: Versuch über den Normalismus, besonders die Teile I bis IV sowie VII bis X.

1135 Zum Zusammenhang von Literatur und Krankheit liegt mittlerweile eine schier unüberschaubare Fülle an Forschungsbeiträgen vor. Wichtige Initialimpulse für die Literatur-und- 
Versuchs hin, Gesundheit allgemeingültig zu normieren: „Natürlich kann man auch Standardwerte für die Gesundheit festlegen. Wenn man aber etwa einem gesunden Menschen diese Standardwerte aufzwingen wollte, würde man ihn eher krank machen. Es liegt eben im Wesen der Gesundheit, daß sie sich in ihren eigenen Maßen selbst erhält. “1136 Genau eine solche Form des flexiblen Normalismus im Verhältnis zur eigenen Gesundheit wird in der fiktiven Gesellschaft von Corpus Delicti wieder zurückgenommen, wenn die METHODE Gesundheit als allgemeingültigen Wert - im doppelten Sinne einer moralischen Leitlinie und eines nummerischen Standards - definiert und alles, was außerhalb dieses Wertes liegt, für krank - wiederum im doppelten Sinne der Deskription und der moralischen Wertung - erklärt. In einer derartigen Gesellschaft bleibt kein Platz für eine individuelle Behandlung von Gesundheitsfragen; an die Stelle subjektiver Bewertungen des eigenen Gesundheitsempfindens tritt der, so Zeh in einem Artikel für die FAZ, „Zwang zur ,Normalität““. ${ }^{1137}$ Damit werden aber auch die Errungenschaften liberaler Demokratien preisgegeben, welche gerade in der Fähigkeit zur Deliberation und friedlichen Vermittlung divergenter Positionen bestehen. ${ }^{1138}$

Die protonormalistische Gleichschaltung des öffentlichen Diskurses deutet sich in Corpus Delicti bereits über die Titel der öffentlichen Medien an: So heißt eine populäre Fernsehsendung WAS ALLE DENKEN (CD, 83); und das zentrale Publikationsorgan der METHODE trägt den Titel DER GESUNDE MENSCHENVERSTAND. In dieser Titelformulierung werden Vernunft, Gesundheit und Normalität in ein enges Abhängigkeitsverhältnis zueinander gebracht (tatsächlich dient die von Heinrich Kramer verantwortete Zeitschrift nicht zuletzt dem Zweck, Staatspropaganda zu

Krankheit-Forschung lieferte Susan Sonntag: Illness as Metaphor and AIDS and Its Metaphors. London 2002. Siehe zum Zusammenhang von Literatur und Gesundheit jetzt Letizia Dieckmann / Julian Menninger / Michael Navratil (Hg.): Gesundheit erzählen. Ästhetik Performanz - Ideologie. Berlin / Boston 2021 (in Vorbereitung).

1136 Hans-Georg Gadamer: Über die Verborgenheit der Gesundheit. Aufsätze und Vorträge. Frankfurt a. M. 1993, S. 138.

1137 Zeh: Schützt den Datenkörper. Siehe zum Thema der Datenüberwachung in Corpus Delicti auch Koellner: Data, Love, and Bodies.

1138 In ihren Frankfurter Poetikvorlesungen votiert Zeh für ein pluralistisches Verständnis von Normalität: „Normalität ist eine Absprache von mindestens zwei, am besten zwischen erheblich mehr Personen. Eine Gesellschaft, die nur eine Normalität kennt, ist totalitär. Wir brauchen möglichst zahlreiche, möglichst unterschiedliche, sich überlagernde, changierende Normalitätsabsprachen, ein wahres Schichtenmodell aus Normalitäten, um es möglichst vielen Menschen zu ermöglichen, sich einigermaßen geistig gesund zu fühlen." (Zeh: Treideln, S. 148). 
verbreiten und politische Gegner öffentlich zu diffamieren). Die ideale Geliebte Mias imaginäre Gesprächspartnerin und die Verteidigerin von Moritz' oppositionellen Ansichten nach dessen Tod - wendet sich gegen diese suggestive Sprachverwendung, wenn sie behauptet: „Gesunder Menschenverstand [...] ist, wenn einer recht haben will und nicht begründen kann, warum!“ (CD, 37) Damit wird im Roman ein Argument der romantischen Philosophie aufgegriffen, welche den ,gesunden Menschenverstand“ bereits um 1800 unter Ideologieverdacht gestellt hatte. So setzt beispielsweise Hegel den ,gesunden Menschenverstand gerade in Opposition zur wahren Philosophie. ${ }^{1139}$ Angriffe auf den ,gesunden Menschenverstand' finden sich in der Philosophie des 19. Jahrhunderts - etwa bei Marx, Schopenhauer und Nietzsche - dann immer wieder. ${ }^{1140}$ Es erscheint mithin durchaus konsequent, wenn Mias Bruder Moritz, ein entschiedener Gegner der METHODE und ihrer vermeintlich streng rationalen Weltanschauung, ausgerechnet Philosophie studiert. Überhaupt wird im Roman das philosophische, kritische oder auch künstlerische Denken immer wieder in Opposition zum protonormalistischen Konsens des herrschenden Systems gebracht, ohne dass dieser Konsens dadurch jedoch nachhaltig erschüttert werden könnte. ${ }^{1141}$

1139 Bereits in der Differenzschrift sieht Hegel den ,gesunden Menschenverstand“ als der philosophischen Spekulation unterlegen an: „Die Spekulation versteht deswegen den gesunden Menschenverstand wohl, aber der gesunde Menschenverstand nicht das Tun der Spekulation.“ (Georg Wilhelm Friedrich Hegel: Differenz des Fichteschen und des Schellingschen Systems der Philosophie (1801). In: Ders.: Jenaer Schriften 1801-1807. Werke 2. Frankfurt a. M. 1986, S. 7-136, hier S. 13) In der Phänomenologie des Geistes spitzt Hegel seine Kritik am ,gesunden Menschenverstand“ dann polemisch zu: „Dagegen im ruhigeren Bette des gesunden Menschenverstandes fortfließend, gibt das natürliche Philosophieren eine Rhetorik trivialer Wahrheiten zum besten. Wird ihm die Unbedeutendheit derselben vorgehalten, so versichert es dagegen, daß der Sinn und die Erfüllung in seinem Herzen vorhanden sei, und auch so bei anderen vorhanden sein müsse, indem es überhaupt mit der Unschuld des Herzens und der Reinheit des Gewissens und dgl. letzte Dinge gesagt zu haben meint, wogegen weder Einrede stattfinde noch etwas weiteres gefordert werden könne.“ (Georg Wilhelm Friedrich Hegel: Phänomenologie des Geistes. Werke 3. Frankfurt a. M. 1986, S. 64).

1140 Vgl. F. Vonessen: Gesund, Gesundheit. In: Joachim Ritter (Hg.): Historisches Wörterbuch der Philosophie. Basel 1974, Bd. 3, S. 559-561, hier S. 560.

1141 Die ideale Geliebte besteht aus „Kupferrohre[n]“, die „,beliebig miteinander [...] verschweiß[t]“ sind, befindet sich „an einem Kreuzungspunkt zwischen den Welten“ und stellt etwas dezidiert „Zweckloses“ (CD, 25) dar. Man könnte die ideale Geliebte entsprechend als Allegorie künstlerischen Denkens überhaupt ansehen. Auch bezeichnet Kramer Mia nach einem Streitgespräch als „Dichterin“ und bittet darum, sie „zitieren“ zu dürfen (CD, 125) (allerdings nur, um diese Zitate dann in der Hetzkampagne gegen sie zu verwenden). Schließlich finden sich in Mias Bücherregal Werke von Rousseau (der die Bedeutung von Natürlichkeit und individueller Freiheit gegenüber den zersetzenden Wirkungen der Zivilisation hervorhob), 
Verstöße gegen den, wenn man so will, neo-protonormalistischen Konsens werden in Zehs Roman mit dem Ausschluss aus der Gemeinschaft geahndet. „Die Methode“, so erklärt Kramer, „definiert die Übereinstimmung von allgemeinem und persönlichem Wohl als ,normal'. Wer sich selbst nicht als normal in diesem Sinne begreift, wird es auch in den Augen der Gesellschaft nicht sein. Außerhalb der Normalität herrscht Einsamkeit.“ (CD, 87) Indem Mia die Grundsätze der METHODE in Zweifel zieht, gerät sie in eine Außenseiterposition, die vonseiten des Staates mit der strukturellen Funktion der Terroristin belegt und darüber hinaus metaphorisch mit dem Bild der ,Hexe` in Verbindung gebracht wird.

\subsubsection{Die Wiederkehr des Mittelalters in der Zukunft}

In einer Gesellschaft, die nur einen einzigen Normalitätswert akzeptiert, führt nicht erst die offene Opposition zu politischen oder gesellschaftlichen Irritationen, sondern bereits die bloße „Ambivalenz“ (CD, 126) gegenüber der herrschenden Ordnung: „Noch hat keiner Mia als ,unnormal‘ bezeichnet. Aber auch ,normal' würde sie niemand nennen. Sie sitzt auf dem Zaun.“ (CD, 146) Diese metaphorische Ausdrucksweise verweist auf eine zentrale Passage des Romans, in welcher die ideale Geliebten Mia die Etymologie des Wortes ,Hexe“ auseinandersetzt:

Das Wort kommt von Hagazussa. Die Hexe ist ein Heckengeist. Ein Wesen, das auf Zäunen lebt. Der Besen war ursprünglich eine gegabelte Zaunstange. [...] Zäune und Hecken sind Grenzen, Mia. Die Zaunreiterin befindet sich auf der Grenze zwischen Zivilisation und Wildnis. Zwischen Diesseits und Jenseits, Leben und Tod, Körper und Geist. Zwischen Ja und Nein, Glaube und Atheismus. Sie weiß nicht, zu welcher Seite sie gehört. Ihr Reich ist das Dazwischen.

(CD, 144)

Orwell (der mit seinem dystopischen Roman 1984 zweifellos die kanonische Dystopie zum Thema staatliche Überwachung verfasst hat) und Agamben (der Foucaults Thesen zur Biopolitik weiterentwickelte und darüber hinaus über den „Ausnahmezustand“ (vgl. auch CD, 206) als Modus der Unrechtspolitik philosophiert hat); allerdings weist Mia darauf hin, dass sie Agambens Buch - und möglicherweise auch die Werke der anderen genannten Autoren - „nie gelesen“ (CD, 128) habe. Man kann das als Hinweis darauf deuten, dass in einer Welt, in der eine kritische Auseinandersetzung mit den Rechten und Pflichten von Staat, Gesellschaft und Individuum nicht mehr stattfindet - sei es in Form politischer Philosophie wie bei Rousseau oder Agamben, sei es in Form dystopischer Literatur wie bei Orwell oder auch, metareflexiv gewendet, bei Zeh selbst -, der Entwicklung der politischen Ordnung hin zu einer Diktatur keine Schranken gesetzt sind. 
Auf die politische Bedeutung des „Dazwischen“ wird im folgenden Kapitel noch ausführlich einzugehen sein. Zunächst lohnt es jedoch, dem Themenfeld der „Hexenjagd“ (CD, 252) im Roman genauer nachzuspüren. ${ }^{1142}$

Die Hexenverfolgung der Frühen Neuzeit (einer weit verbreiteten Übergeneralisierung gemäß wird im Roman nicht zwischen Früher Neuzeit und Mittelalter unterschieden) bildet eine der zentralen realweltlichen Bezugsebenen des Romans. Die Forschung hat eine ganze Reihe von Beziehungen zwischen Zehs Roman und den frühneuzeitlichen Hexenprozessen herausgearbeitet. Insbesondere Sonja E. Klocke stellt in einem materialreichen Artikel die einschlägigen Verbindungen zwischen fiktionaler Welt und realer Hexenverfolgung heraus. ${ }^{1143}$ So haben sowohl Mia Holl als auch Heinrich Kramer reale Vorgänger aus dem historischen Kontext der Hexenverfolgung. Insofern, als die Biografien dieser realhistorischen Personen durch die Figuren des Romans in signifikanter Weise variiert werden, können letztere als kontrafaktische Wiedergänger der frühneuzeitlichen Personen angesehen werden.

Der Name der Protagonistin Mia Holl verweist auf Maria Holl (ca. 1549-1634), eine Gastwirtin aus Nördlingen, die 1593 als Hexe inhaftiert wurde und 62 Folterungen über sich ergehen lassen musste. Da Maria Holl allerdings standhaft an ihrer Unschuldsbehauptung festhielt und auch keine weiteren Frauen der Hexerei bezichtigte, wurde sie schließlich wieder auf freien Fuß gesetzt. ${ }^{1144}$ Auch Mia Holl in Corpus Delicti weigert sich, eine Falschaussage zu machen, obwohl ihr die Folter angedroht und schließlich auch an ihr vollstreckt wird. Die finale ,Freilassung' bedeutet im Falle Mias, anders als im Falle ihres realweltlichen Vorbilds, jedoch gerade keinen Schritt in die tatsächliche Freiheit, sondern bildet als Reintegration in eine totalitäre Gesellschaft gerade eine besonders krasse Form der Unterdrückung. Mias Bruder, der im Gefängnis Selbstmord begeht, konnte noch behaupten: „Wer stirbt, entwischt. Wer eingefroren wird, gehört endgültig dem System. Als Jagdtrophäe.“ (CD, 231) Das Einfrieren auf unbestimmte Zeit, zu dem Mia am Ende des Schauprozesses verurteilt wird, bedeutet zwar nicht im eigentlichen Sinne eine Todesstrafe (die ein System, welches „sich auf eine absolute

1142 Mögliche intertextuelle Verbindungen zwischen Corpus Delicti und Arthur Millers Stück Hexenjagd (im Original: The Crucible (1953)) diskutieren Schotte und Vorbeck-Heyn: Die Zukunft unserer Gesellschaft liegt in ihrer Vergangenheit, S. 119, Anm. 9.

1143 Sonja E. Klocke: „Das Mittelalter ist keine Epoche. Mittelalter ist der Name der menschlichen Natur.“ Aufstörung, Verstörung und Entstörung in Juli Zehs „Corpus Delicti“. In: Carsten Gansel / Norman Ächtler (Hg.): Das ,Prinzip Störung“ in den Geistes- und Sozialwissenschaften. Berlin / Boston 2013, S. 185-202. Siehe auch Schotte / Vorbeck-Heyn: Die Zukunft unserer Gesellschaft liegt in ihrer Vergangenheit.

1144 Vgl. Lyndal Roper: Hexenwahn. Geschichte einer Verfolgung. München 2007, S. 78, S. 377 f., Anm. 14. 
Wertschätzung des menschlichen Lebens stützt“ (CD, 231), auch gar nicht verhängen könnte $\left.{ }^{1145}\right)$. Für das Individuum sind Tod und Einfrieren aber letztlich fast gleichbedeutend (man kann im Einfrieren eine genaue Umkehrung des Feuertodes auf dem Scheiterhaufen erkennen ${ }^{1146}$ ). Beide Formen der Beseitigung politischer Gegner würden aber immerhin die Möglichkeit eröffnen, zumindest symbolisch gegen das herrschende System in Stellung gebracht zu werden. Genau eine solche Verklärung als „Märtyrerin“ (CD, 263) enthält die Methode Mia Holl vor, wenn in letzter Minute eine „Begnadigung der Verurteilten“ (CD, 263) ausgesprochen wird, eine Begnadigung jedoch, welche sich anders als im Fall der realhistorischen Maria Holl, die bis zum heutigen Tag als Lokalheldin verehrt wird ${ }^{1147}$ - nicht als Erfolgsgeschichte vom Widerstand des Individuums gegenüber den Zugriffen der Obrigkeit symbolisch instrumentalisieren lässt, sondern die im Gegenteil gerade die totale Macht der Obrigkeit demonstriert. Mia Holl wird, so bemerkt Heinz-Peter Preußer, „keine Jeanne d'Arc werden, auch keine Antigone oder Ulrike Meinhof, sondern in die psychologische Betreuung abgeschoben“. ${ }^{1148}$ Anlässlich von Mias Freilassung sinniert Heinrich Kramer:
„[N]ur unfähige Machthaber [schenken] dem nervösen Volk eine Kultfigur. Jesus von Na- zareth, Jeanne d'Arc - der Tod verleiht dem Einzelnen Unsterblichkeit und stärkt die Kräfte des Widerstands. Das wird Ihnen nicht passieren, Frau Holl. Stehen Sie auf. Ziehen Sie sich an. Gehen Sie nach Hause. Sie sind ...“ Noch einmal kehrt der Lachanfall zurück. „Frei!“
(CD, 363f.)

Heinrich Kramer wiederum verdankt seinen Namen dem Dominikaner-Mönch Heinrich Kramer (Institoris) (ca. 1430-1505), welcher im Jahre 1486 den berüchtigten Malleus Maleficarum, den Hexenhammer veröffentlichte, die bedeutendste frühneuzeitliche Schrift zur Legitimation der Hexenverfolgung. Ein Verweis auf den Hexenhammer findet sich bereits zu Beginn von Zehs Roman, wenn, wie

1145 Bereits Foucault macht darauf aufmerksam, dass eine biopolitisch orientierte Macht, wie sie sich in der Moderne zusehends etabliert, auf die Todesstrafe weitgehend verzichten muss, da sich die Autorität dieser Macht ja gerade auf eine Verwaltung des Lebens stützt: „Seit die Macht das Leben in seine Regie genommen hat, ist die Anwendung der Todesstrafe nicht durch humanitäre Gefühle, sondern durch die innere Existenzberechtigung der Macht und die Logik ihrer Ausübung immer mehr erschwert worden. Wie sollte eine Macht ihr höchstes Vorrecht in der Verhängung des Todes äußern, wenn ihre Hauptaufgabe darin besteht, das Leben zu sichern, zu verteidigen, zu stärken, zu mehren und zu ordnen?“ (Foucault: Der Wille zum Wissen, S. 133).

1146 Vgl. Klocke: „Das Mittelalter ist keine Epoche“, S. 196.

1147 Vgl. Roper: Hexenwahn, S. 7 f.

1148 Preußer: Gewalt und Überwachung, S. 182. 
oben ausgeführt, extensiv aus Heinrich Kramers Schrift Gesundheit als Prinzip staatlicher Legitimation zitiert wird. ${ }^{1149}$ Ebenso wie der realhistorische Hexenhammer liefert die Schrift des fiktiven Heinrich Kramer eine ideologische Rechtfertigung für die Ausgrenzung und Verfolgung Andersdenkender; auch erscheinen beide Werke in hoher Auflage. ${ }^{1150}$ Anspielungen auf die frühneuzeitliche Legitimationsschrift der Hexenverfolgung innerhalb von Zehs Roman verweisen dabei niemals nur auf einen bestimmen Prätext, sondern deuten zugleich auch auf die weitreichenden gesellschaftlichen Folgen hin, welche die Veröffentlichung dieses Werkes nach sich zog: Wohl kaum eine Buchpublikation der Frühen Neuzeit dürfte ähnlich fatale Auswirkungen in der realen Welt gezeitigt haben wie der Hexenhammer. ${ }^{1151}$ Die intertextuellen Bezüge auf den Hexenhammer in Zehs Roman liefern insofern auch immer indirekte transfiktionale (kontrafaktische) Bezüge zur realen Hexenverfolgung, welche durch den Hexenhammer befeuert wurde. Indem Kramers Werk in Corpus Delicti als kontrafaktischer Hexenhammer erscheint, wird die ideologisch-propagandistische Funktion seiner Gesundheitsschrift überdeutlich herausgestellt.

Wichtigstes sachliches Verbindungsglied zwischen der fiktionalen Welt und der Hexenverfolgung bildet die Folter, durch die Mia dazu gebracht werden soll, ihre Mitgliedschaft in einer terroristischen Vereinigung einzugestehen. Im Verweis auf das Thema Folter werden dabei nicht nur Vergangenheit und projizierte Zukunft in Beziehung zueinander gesetzt; es eröffnen sich darüber hinaus Bezüge auf die Gegenwart der Textentstehung zu Beginn des 21. Jahrhunderts. So lässt Kramer Mia wissen:

An den technischen Details hat sich wenig geändert. Da funktioniert im Wesentlichen alles wie vor fünfzig Jahren. Man stellt Sie auf eine Kiste, nackt, versteht sich, und zieht Ihnen eine schwarze Kapuze über den Kopf. An ihren Fingern, Zehen und primären Geschlechtsteilen werden Kontakte befestigt, Wäscheklammern nicht unähnlich. [...] Die Stromstärke wird stufenlos hochgefahren. Zwei gut ausgebildete Ärzte vom Universitätsklinikum sorgen dafür, dass Sie nicht ... draufgehen.

(CD, 235)

$1149 \mathrm{Zu}$ Ideen des Hexenhammers, die in Corpus Delicti anklingen, siehe Klocke: „Das Mittelalter ist keine Epoche“, S. 192-197.

1150 Bis zum Ende des 17. Jahrhunderts wurden über 30 Auflagen des Hexenhammers gedruckt, etwa 30.000 Exemplare waren in Europa in Zirkulation. Vgl. Tamar Herzig: Witches, Saints, and Heretics. Heinrich Kramer’s Ties with Italian Women Mystics. In: Magic, Ritual, and Witchcraft 1/1 (2006), S. 26-55, hier S. 27.

1151 Siehe zur Rezeption des Malleus Maleficarum Günter Jerouschek / Wolfgang Behringer: „Das unheilvollste Buch der Weltliteratur“? Zur Entstehungs- und Wirkungsgeschichte des Malleus Maleficarum und zu den Anfängen der Hexenverfolgung. In: Heinrich Kramer (Institoris): Der Hexenhammer: Malleus Maleficarum. Kommentierte Neuübersetzung. München 2000, S. 9-98. 
Offenkundig wird hier auf den Abu Ghuraib-Folterskandal angespielt: Während der amerikanischen Besatzung des Irak wurden Gefangene im Abu Ghuraib-Gefängnis gefoltert, misshandelt und getötet, was in den Jahren 2004 und 2006 durch die Veröffentlichung einer Reihe spektakulärer Fotos publik wurde. Das bekannteste dieser Fotos zeigt einen irakischen Gefangenen, der mit ausgebreiteten Armen und an eine Reihe stromführender Drähte angeschlossen auf einer Kiste steht, eine Kapuze über dem Kopf. ${ }^{1152}$ Auch jenseits ihrer konkreten politischen Brisanz haben sich die Vorfälle von Abu Ghuraib ins kollektive Gedächtnis eingebrannt als Symbol für eine Wiederkehr politischer Handlungsweisen, welche zu Beginn des 21. Jahrhunderts eigentlich als zivilisatorisch überwunden gegolten hatten. Indem das vermeintlich ideale Staatssystem in Zehs Roman auf das Instrument der Folter zurückgreift, verweist Corpus Delicti zugleich auf die Bedrohung der Menschenrechte in der Gegenwart der Leserschaft und betont damit die prinzipielle Fragilität zivilisatorischer Errungenschaften. So kommt Mia, nachdem ihr von Heinrich Kramer die Folter angedroht wurde, zu dem pessimistischen Schluss: „Es hat sich nichts geändert. Es ändert sich niemals etwas. Ein System ist so gut wie das andere. Das Mittelalter ist keine Epoche. Mittelalter ist der Name der menschlichen Natur.“(CD, 235)

Über das Thema Folter hinaus eröffnet die Anspielung auf den Abu Ghuraib-Skandal Verbindungen zum Thema Terrorismus, dem im Roman generell zentrale Bedeutung zukommt. Ebenso wie in der Streitschrift Angriff auf die Freiheit oder Zehs Stück Der Kaktus, das die Anwendung gewaltsamer Verhörmethoden gegen einen Terrorverdächtigen thematisiert, steht dabei in Corpus Delicti weniger der Terrorismus an sich zur Diskussion - etwa die ihm zugrundeliegenden Motivationen, seine (Il-)Legitimität oder das Weltbild der ausführenden Terroristen -, sondern vielmehr das Verhalten derjenigen Akteure, die sich entweder vom Terrorismus bedroht fühlen oder aber die das Schreckgespenst des Terrorismus zur Durchsetzung eigener politischer Anliegen heraufbeschwören - und damit vorauseilend genau jene Freiheiten zerstören, die im Kampf gegen den Terrorismus angeblich verteidigt werden sollen. ${ }^{1153}$ Reale Ter-

1152 Vgl. Klocke: „Das Mittelalter ist keine Epoche“, S. 197 f. Siehe auch Müller-Dietz: Strafrecht im Zukunftsstaat?, S. 438.

1153 Das Thema Terrorismus spielt bereits in Zehs zweitem Roman Spieltrieb eine Rolle. Vgl. etwa Juli Zeh: Spieltrieb. München ${ }^{9} 2006$, S. 146-151. Die Möglichkeit eines Staatsumsturzes mit gewaltsamen Mitteln thematisiert Zeh dann in ihrem zweiten dystopischen Roman Leere Herzen (übrigens ebenfalls unter Verweis auf die RAF). Die Möglichkeit eines solchen Umsturzes wird am Ende dieses Romans entschieden zurückgewiesen. Vgl. Juli Zeh: Leere Herzen. München 2017, S. 346f. Ein wichtiger Unterschied zwischen der autoritären Regierung in Corpus Delicti und derjenigen in Leere Herzen besteht allerdings darin, dass die letztere demokratisch gewählt ist, während die Art der Regierungskonstitution in Corpus Delicti nicht thema- 
roristen kommen in Corpus Delicti überhaupt nicht vor, sodass es auch wenig plausibel erscheint, etwaigen Verbindungen zwischen Mia Holl und den Terroristen der Baader-Meinhof-Gruppe nachzuspüren. ${ }^{1154}$ Zwar spielt der Name der Gruppe „Recht auf Krankheit“ (R.A.K) (CD, 83) im Roman erkennbar auf die terroristische Vereinigung „Rote Armee Fraktion“ (RAF) an. Allerdings bleibt bis zum Ende des Romans unklar, ob diese Gruppe überhaupt existiert. Auch wird im Text mehrfach betont, dass sowohl Mia als auch ihr Bruder Moritz, ungeachtet ihrer oppositionellen Haltung gegenüber der METHODE, keinerlei Sympathien für etwaige Terrorgruppen hegen (vgl. CD, 148f., 196). Wenn Mia Holl mit dem Terrorismus assoziiert wird, so nicht deshalb, weil sie im Roman als Ulrike Meinhof des 21. Jahrhunderts erschiene ${ }^{1155}$, sondern vielmehr deshalb, weil hier die gesellschaftlich-politische Funktion des Labels ,Terrorist" kenntlich gemacht wird - auch und gerade dort, wo eine reale Bedrohung gar nicht besteht. (Eher noch als auf die RAF-Terroristen wird auf die bürgerrechtseinschränkenden Maßnahmen verwiesen, die seinerzeit im Kampf gegen die RAF-Terroristen sowie gegen vermeintliche RAF-Sympathisanten umgesetzt wurden. ${ }^{1156}$ ) Das Label ,Terrorist“ erfüllt im Roman - und, so wird durch die Verweise auf die Gegenwart der Leser suggeriert, auch darüber hinaus - eine ähnliche Funktion wie das Label ,Hexe‘ in der Frühen Neuzeit: Beide dienen der Konstruktion einer imaginären Bedrohung sowie der Legitimierung von Repressalien gegenüber einzelnen Mitgliedern der Bevölkerung.

Neben der frühneuzeitlichen Hexenverfolgung, welche in Corpus Delicti in Form kontrafaktischer Variationen aufgerufen wird, und den zeitgenössischen Diskursen der Biopolitik, des Terrorismus und der Folter, die vor allem in Form von Anspielungen aufscheinen, greift Juli Zeh in ihrem Roman noch auf eine weitere bedeutende Quelle realweltlichen Faktenmaterials zurück: nämlich auf die eigene Person. Es können eine ganze Reihe formaler Auffälligkeiten benannt werden, welche eine transfiktionale Verbindung von fiktiven Figuren mit der empirischen Autorin nahelegen, insbesondere im Falle der Protagonistin Mia Holl. Bereits die leichte Abwandlung des Namens der realhistorischen Person

tisiert wird. Im Interview hat Zeh das Recht auf gewaltsamen Widerstand innerhalb totalitärer Systeme verteidigt. Vgl. Philipp Schwenke / Juli Zeh: „Uns fehlen die Parolen“. In: Zeit Campus, 11.09.2008. Siehe auch Borgans / Meißner / Zeh: „Ich bin ein großer Fan der Freiheit“.

1154 Vgl. Schotte / Vorbeck-Heyn: Die Zukunft unserer Gesellschaft liegt in ihrer Vergangenheit, S. 125-128.

1155 Dass Mia Holl, wie Schotte und Vorbeck-Heyn behaupten, „unverkennbar Züge Ulrike Meinhofs [trage]“, erscheint mir wenig überzeugend. Vgl. Schotte / Vorbeck-Heyn: Die Zukunft unserer Gesellschaft liegt in ihrer Vergangenheit, S. 125.

1156 Vgl. McCalmont / Maierhofer: Juli Zeh’s Corpus Delicti (2009), S. 181 f.; siehe auch Trojanow / Zeh: Angriff auf die Freiheit, S. 65-72. 
Maria Holl zu Mia Holl lässt - rein phonotaktisch betrachtet - eine Assoziation der Protagonistin des Romans mit seiner Autorin Juli Zeh als naheliegend erscheinen. Auch der Umstand, dass der Name ,Mia“ in mehreren romanischen Sprachen dem Possessivpronomen ,mein/meine“ entspricht, deutet auf eine Teilidentität zwischen der Autorin und ihrer Protagonistin hin. ${ }^{1157}$ In der Theaterfassung von Corpus Delicti aus dem Jahre 2007 ergibt sich ein starker autofiktionaler Verweis auf die Autorin Zeh darüber hinaus durch das Geburtsjahr der Protagonistin im Jahr 2024, welches genau fünfzig Jahre nach Juli Zehs eigenem Geburtsjahr 1974 liegt (während die Handlung des Stückes im Jahr 2057, also wiederum genau fünfzig Jahre nach der Uraufführung von Zehs Stück, angesiedelt ist). ${ }^{1158}$ Noch deutlicher, wenn auch medial anders gelagert, fallen die autofiktionalen Verweise in der als „Schallnovelle“ untertitelten Hörfassung des Stoffes aus, die Juli Zeh zusammen mit der Band Slut produziert hat und die ein halbes Jahr nach Erscheinen des Romans, im Herbst 2009, auf den Markt kam: In dieser Fassung spricht Zeh selbst die Rolle der Mia Holl. Auf einer Tour mit dem Schallnovellen-Projekt im selben Jahr war Zeh auch als Schauspielerin auf der Bühne zu sehen, sodass eine direkte performative Identifikation von Autorin und Figur möglich wurde.

Gewissermaßen über Bande gespielt wird der autofiktionale Verweis im Falle einer weiteren Romanfigur: der jungen Richterin Sophie. Anhand der juristischen Karriere wird auch bei dieser Figur eine autofiktionale Beziehung zur Biografie der Autorin Zeh etabliert. Zugleich fungiert die Richterin, so bemerkt Achim Geisenhanslüke, als „eine[...] Art Zwillingsfigur der Hauptfigur Mia Holl.“1159 Sophie vertritt als Richterin zwar die Interessen der METHODE, bildet unter den Organen staatlicher Gewalt in Corpus Delicti jedoch eher eine begütigend-ausgleichende Stimme. Als eine Art Neben-Mia tritt auch Sophie im Roman zunächst als treue Anhängerin der METHODE auf, wird im Laufe der Handlung dann aber immer stärker in eine Außenseiterposition gedrängt und kommt ab einem gewissen Punkt gar nicht mehr vor: Als Mia durch die Aufdeckung der

1157 Vgl. Layh: Finstere neue Welten, S. 172.

1158 Einen vergleichbaren, wenn auch weniger deutlichen Verweis auf den Publikationszeitpunkt des Romans im Jahre 2009 lässt sich in dem Detail erkennen, dass die erste Vorverhandlung gegen Mia Holl im „Raum 20/09“ (CD, 12) stattfindet. Auch erwähnt Kramer in abschreckender Absicht die „Sterbestatistiken aus dem Jahr 2009“ (CD, 85). Im Nebentext der Dramenfassung wird darüber hinaus vorgeschlagen, „das Datum des Urteils gegen Mia Holl, das zu Beginn der Handlung in der erzählten Zeit des Jahres 2057 verkündet wird, mit Tag und Monat der jeweiligen Aufführung anzugeben." (Weitin: Ermittlung der Gegenwart, S. 71) Für einen Vergleich von Dramen- und Romanfassung siehe Schönfellner: Die Perfektionierbarkeit des Menschen?, S. 75-77.

1159 Geisenhanslüke: Die verlorene Ehre der Mia Holl, S. 227. 
Unschuld ihres Bruders in offene Opposition zur METHODE gerät, wird Sophie durch einen anderen Richter ersetzt. ${ }^{1160}$

Für die autofiktionale Dimension von Corpus Delicti besonders aufschlussreich ist der verlegerische Peritext: Auf der Rückseite der gebundenen Ausgabe des Romans ist ein Foto von Juli Zeh zu sehen, die sich an eine über einem Maschendrahtzaun verlaufende Stange anlehnt. Durch dieses zunächst wenig auffällige Hintergrunddetail wird die Autorin selbst mit der „Zaunreiterin“ (CD, 144) Mia Holl in Verbindung gebracht. ${ }^{1161}$ Als Zaunreiterin verweigert die ,Hexe‘ im Roman gerade eine eindeutige Positionierung - und gerät damit sowohl ins Abseits der Gesellschaft als auch ins Fadenkreuz des Methodenschutzes. „Wer keine Seite wählt“, so stellt die ideale Geliebte im Roman fest, „ist ein Außenseiter. Und Außenseiter leben gefährlich.“ (CD, 144) Als eine der erfolgreichsten deutschsprachigen Autorinnen der Gegenwart kann Juli Zeh selbst zwar kaum als ,Außenseiterin“ betrachtet werden. Gerade ihre deutlichen Positionierungen als engagierte Intellektuelle haben Zeh jedoch immer wieder zur Zielschreibe öffentlicher Anfeindungen werden lassen. So hält Sabrina Wagner fest: „Juli Zeh polarisiert und sieht sich [...] neben überschwänglichem Lob an anderer Stelle mit harter Kritik, die nicht immer sachlich bleibt, konfrontiert." ${ }^{\prime 162}$ Für eine Interpretation von Corpus Delicti sind die Biografie Zehs und die mediale Kritik an ihrer Person zwar nicht im Einzelnen von Interesse - es handelt sich bei Zehs Roman gewiss nicht um eine Autofiktion in dem Sinne, dass die individuelle Biografie der Autorin hier in fiktionalisierter Form präsentiert würde. Auch spricht nichts für die Annahme, Zeh habe sich durch die Verweise auf die eigene Person innerhalb von Corpus Delicti zum singulären Opfer staatlicher Repressalien stilisieren wollen. ${ }^{1163}$ Indem Zeh die Protagonistin ihres Romans als kontrafaktisch-autofiktionale Variante ihrer selbst anlegt, macht sie

1160 Bereits in Zehs Roman Spieltrieb tritt eine Richterin auf, welche „[d]ie kalte Sophie“ genannt wird (Zeh: Spieltrieb, S. 517). Diese Figur weist gleichfalls Gemeinsamkeiten mit der Protagonistin des Romans, der hochbegabten Schülerin Ada, auf. Auch in Spieltrieb lassen sich beide Figuren mit der Autorin Zeh in Verbindung bringen.

1161 Vgl. Schotte / Vorbeck-Heyn: Die Zukunft unserer Gesellschaft liegt in ihrer Vergangenheit, S. $122 \mathrm{f}$.

1162 Wagner: Aufklärer der Gegenwart, S. 69.

1163 In einem Essay mit dem Titel Zur Hölle mit der Authentizität! spricht Zeh sich gegen einen Umgang mit fiktionaler Literatur aus, der allzu unmittelbar nach dem Faktengehalt des Geschriebenen, etwa nach Übernahmen aus der Autorenbiografie, fragt: „Eine Erzählung zu schreiben, die eins zu eins ein tatsächliches Ereignis spiegelt, wäre für mich todlangweilig; das vollständige Erfinden einer Geschichte, die nichts mit mir zu tun hat, hingegen ohne Sinn.“ (Juli Zeh: Zur Hölle mit der Authentizität! In: Die Zeit, 21.09.2006) In einem mittlerweile nicht mehr online verfügbaren Interview mit Ingrid Brodnig hat Zeh sich gegenüber einer he- 
vielmehr ganz prinzipiell darauf aufmerksam, dass sich kritische Intellektuelle in der Gegenwart mitunter harscher Kritik, öffentlichen Anfeindungen oder gar handfesten Drohungen ausgesetzt sehen. In ihren Frankfurter Poetikvorlesungen weist Zeh explizit auf die Diffamierung des engagierten Intellektuellen hin:

Seit unser Land beschlossen hat, sich auf dem Höhepunkt von Wohlstand, Sicherheit, Gesundheit und Frieden zur Krisengesellschaft zu erklären; seit der Winter „Schneechaos“, der Sommer „Jahrhunderthitze“ und starker Regen „Flutkatastrophe“ heißen; seit wir hinter jeder Grippe-Erkrankung eine Pandemie wittern und Kopftücher für eine Bedrohung des Abendlandes halten, bildet der zunehmend homogene Diskurs Abwehrkräfte wie ein Immunsystem, das sich gegen Kritik am Mainstream zur Wehr setzt. Wer wollte schon riskieren, durch das Äußern von Einzelmeinungen zum Opfer einer immunologischen Abwehrreaktion zu werden? $\mathrm{Zu}$ gewinnen gibt es wenig, zu verlieren viel. Am Ende ist man eine lächerliche Figur, steht als Karikatur des engagierten Intellektuellen mutterseelenallein an der medialen Schusslinie und fuchtelt mit der stumpfen Lanze. ${ }^{1164}$

So wie die engagierten Intellektuellen durch die „Abwehrkräfte“ eines „zunehmend homogene[n] Diskurs[es]“ angegriffen werden, so wendet sich auch die „METHODE als Immunsystem des Landes“ gegen das „grassierende Virus“ (CD, 201) des kritischen Denkens, welches in der Imago der ,Hexe“ zur suggestiv-medienwirksamen Repräsentation gelangt. Mia fungiert somit im Roman - nebst vielem anderen - als Vertreterin des „engagierten Intellektuellen“, welcher gerade aufgrund seines Engagements für politische Belange Gefahr läuft, sich ins gesellschaftliche Abseits zu manövrieren und damit in letzter Konsequenz zum Staatsfeind - respektive zum „Methodenfeind“ (CD, 73) - zu werden.

\subsubsection{Das Dazwischen als Raum des Politischen}

In der Forschung wurde immer wieder behauptet, Corpus Delicti weise eine eindeutige politische Botschaft auf: Konstatiert wurde eine „streckenweise thesenhafte“ Gestaltung des Romans ${ }^{1165}$, eine „clear political message“ “1166 oder gar eine „plakative Kritik an gesellschaftlichen Normierungsprozessen“. ${ }^{1167}$ Auch Zeh selbst hat mit ihren öffentlichen Äußerungen im Publikationskontext des Romans (einige wurden zu Beginn des Kapitels zitiert) die Vorstellung nahegelegt,

roisierenden Identifizierung der eigenen Person mit der Protagonistin von Corpus Delicti verwahrt. Vgl. McCalmont / Maierhofer: Juli Zeh’s Corpus Delicti (2009), S. 383.

1164 Zeh: Treideln, S. $159 \mathrm{f}$.

1165 Neuhaus: Konsequenzen der Biopolitik, S. 126.

1166 McCalmont / Maierhofer: Juli Zeh's Corpus Delicti (2009), S. 376.

1167 Schönfellner: Die Perfektionierbarkeit des Menschen?, S. 69. 
in Corpus Delicti werde eine eindeutige politische Botschaft verbreitet, vergleichbar derjenigen in dem Sachbuch Angriff auf die Freiheit oder in den journalistischen Arbeiten der Autorin.

Dass Zeh mit Corpus Delicti gegenüber der Idee einer Absolutsetzung der Gesundheit eine ablehnende Position einnimmt, dürfte tatsächlich nicht zu leugnen sein. Nichtsdestoweniger lohnt es, die formale Gestaltung dieser Ablehnung im Roman, gewissermaßen also die Art und Weise ihrer literarischen Inszenierung, genauer in den Blick zu nehmen. Dabei lässt sich zeigen, dass die vermeintlich so eindeutige Botschaft im Rahmen der literarischen Bearbeitung beträchtlich an Komplexität und Ambivalenz gewinnt. ${ }^{1168}$

Als Vertreter ideologischer Extrempositionen stehen sich im Roman der radikale Individualist Moritz Holl und der METHODEN-Fanatiker Heinrich Kramer gegenüber. ${ }^{1169}$ Nun erscheint es naheliegend, die Position von Moritz schlicht mit der Stimme der Autorin gleichzusetzen und seine diversen Plädoyers - für Individualismus, Freiheit und Liebe - als ausformulierte Botschaften des Romans respektive als Werkintentionen zu deuten, wohingegen die Position Kramers schlicht als totalitär diffamiert wird. Bei genauerer Betrachtung erweist sich eine solche Interpretation allerdings als unterkomplex. Der Roman Corpus Delicti bringt nicht einfach zwei Extrempositionen in Opposition zueinander, sondern führt zugleich das immanente Scheitern dieser Extrempositionen und zwar beider! - vor, da sie gerade aufgrund ihrer Rigorosität letztlich unausweilich in Widerspruch zu sich selbst geraten. So wird Moritz' Totalopposition gegenüber der METHODE durch den Umstand relativiert, dass er sein eigenes Leben der METHODE verdankt, welche ihn als Kind von einer lebensbedrohlichen Leukämieerkrankung geheilt hat. Seine Ablehnung gegenüber der Körperfixierung der METHODE kann Moritz mithin überhaupt nur formulieren, weil eben diese Körperfixierung ihn vor dem Tod bewahrt hat (wobei es dann allerdings wie bittere Ironie anmutet, dass just dieselbe Körperfixierung zu dem Justizirrtum führt, der Moritz schlussendlich in den Tod treibt). Von Kramer wiederum wird behauptet, er denke und spreche „mit einer Rücksichtslosigkeit, die darauf

1168 Dass die Übersetzung politischer Thesen ins Medium der Literatur eine - möglicherweise ungeahnte - Komplexitätssteigerung zur Folge hat, ist auf einer höheren Ebene durchaus mit Zehs Poetik vereinbar. Gerade die Ambivalenz und Unverfügbarkeit von Bedeutung hat Zeh immer wieder zum zentralen Merkmal fiktionaler Literatur erklärt: „Wozu gäbe es denn die ganze Literaturwissenschaft, wenn die Autoren selbst wüssten, was es mit ihren Texten auf sich hat?" (Zeh: Treideln, S. 18).

1169 In seiner Funktion als Sprachrohr der METHodE weist Kramer deutliche Parallelen zu anderen dystopischen ,Erklärerfiguren“ auf, etwa O’Brien in George Orwells 1984 oder Mustapha Mond in Aldous Huxleys Brave New World. Vgl. Schroth: "Bedrohung verlangt Wachsamkeit”, S. 126f.; Neuhaus: Konsequenzen der Biopolitik, S. $126 \mathrm{f}$. 
verzichtet, der ewigen Unentschiedenheit des Menschen auf dialektische Art zur Legitimation zu verhelfen.“ (CD, $126 \mathrm{f}$.) Genau diese totale Abwehr jedweder Ambivalenz erscheint in Corpus Delicti jedoch als eine problematische, ja verwerfliche (wenn auch überaus attraktive) Form des Komplexitätsreduktion: ${ }^{1170}$ Am Beispiel Kramer führt der Roman vor, dass ein System, welches die Gesundheit zum absoluten Wert erhebt, eben zur Verteidigung dieses absoluten Wertes letztlich die Gesundheit selbst opfern wird. ${ }^{1171}$ So offenbart sich das realpolitische Kalkül des „Fanatiker[s]“ (CD, 245) Kramer insbesondere in seiner Bereitschaft, zum Zweck eines Erhalts des herrschenden Systems die Grundsätze der METHODE selbst außer Kraft zu setzen: Der Schauprozess gegen Mia Holl, der Versuch, sie als Mitglied einer (möglicherweise fiktiven) terroristischen Vereinigung zu verleumden sowie die auf gefälschten Indizien beruhende Anschuldigung, Mia habe einen terroristischen Anschlag geplant, laufen den Grundprinzipien der METHODE - Vernunft, Evidenz und objektive Wahrheit - gerade zuwider. Besonders augenfällig wird diese Selbstanullierung der Prinzipien der METHODE bei der Anwendung der Folter an Mia: Im vermeintlichen Dienst der Gesundheit aller wird hier die Gesundheit des Einzelnen willentlich zerstört. Wenn Kramer all diese Vorgehensweisen gegen Mia billigt oder sogar aktiv befördert, so geschieht dies offenkundig nicht zum Zweck einer Verteidigung der Prinzipien der METHODE, sondern allein zu dem Zweck, deren totalitären Geltungs- und Machtanspruch zu sichern. Gipfelpunkt dieses pragmatischen Selbstwiderspruchs ist die letzte Szene des Romans, in welcher Mia, die soeben in die Scheinfreiheit entlassen wurde, von Kramer ,sein Zigarettenetui und das Feuerzeug“ (CD, 264) - also streng verbotene Substanzen - zugeworfen be-

1170 Es wird im Roman verschiedentlich angedeutet, dass die beeindruckende Selbstverständlichkeit, Eleganz und Konsequenz, mit der Kramer die Grundsätze der METHODE propagiert, letztlich Fassade sind. Bereits bei seinem ersten Auftreten im Roman wird betont: „Nur wer Heinrich Kramer besser kennt, weiß, dass er unruhige Finger hat, deren Zittern er gern verbirgt, indem er die Hände in die Hosentaschen schiebt.“ (CD, 15) Im Kapitel „Ambivalenz“ wird dann darauf hingewiesen, dass Kramer unter leicht gewandelten Bedingungen durchaus lächerlich erscheinen mag: „Man könnte vom selben Ausgangspunkt andere Argumente aufeinanderstapeln, könnte wie beim Schach die Farbe wechseln. Dann wäre Kramer keine Ikone der Unbedingtheit, sondern bloß ein mächtiges Streben mit einer leeren Mitte. Ein Schnüffler. Eine lächerliche Figur.“ (CD, 128).

1171 Hierin lässt sich eine Variante des Umschlags totalen Sicherheitsstrebens in Unfreiheit und damit letztlich auch in einen Verlust der Sicherheit selbst - erkennen. Dem ersten Kapitel von Angriff auf die Freiheit haben Trojanow und Zeh ein Zitat von Benjamin Franklin vorangestellt, das just diese dialektische Beziehung thematisiert: „Wer die Freiheit aufgibt, um Sicherheit zu gewinnen, der wird am Ende beides verlieren." (Trojanow / Zeh: Angriff auf die Freiheit, S. 11). 
kommt. Abschließend wird hier überdeutlich herausgestellt, dass Verstöße des Einzelnen gegen die Grundsätze der METHODE letztlich bedeutungslos sind, solange diese Verstöße nur nicht den Fortbestand des diktatorischen Systems gefährden. Heinrich Kramer, der Chefideologe der METHODE, erweist sich vollends als Zyniker der Macht, wenn er Mia, die mittlerweile durch Folter und öffentliche Ächtung körperlich gebrochen und sozial vernichtet ist, genau eines jener Laster wider die Gesundheit anempfiehlt, aufgrund derer Mia ursprünglich in Konflikt mit den Staatsautoritäten geraten war. Oberstes Interesse der Gesundheitsdiktatur ist offenkundig die Selbsterhaltung des Systems - ein Ziel, dem nötigenfalls die Gesundheit selbst geopfert wird.

Einen Gegenentwurf zum Dogmatismus Moritz Holls und Heinrich Kramers führt der Roman anhand der Figur Mia Holl vor, die zwischen dem rationalistischen Prinzip der METHODE und der individualistisch-affektbasierten Position ihres Bruders hin- und hergerissen wird. (Ausdruck dieser Zerrissenheit ist nicht zuletzt Mias emotionales Verhältnis Heinrich Kramer gegenüber, welches zwischen Hass und erotischer Attraktion oszilliert.) Bei dem „Prozess“ im Untertitel des Romans handelt es sich insofern um mehr als eine Betonung der juristischen Dimension des Textes - also des „Strafprozess[es]“ (CD, 67) gegen Mia Holl - oder einen Verweis auf Franz Kafkas gleichnamigen Roman. ${ }^{1172}$ Der Untertitel bezeichnet eben auch, wie Christopher Schmidt es formuliert, den „schmerzhafte[n] Prozess der politischen Bewusstwerdung“. ${ }^{1173}$ Dieser Prozess schreitet notwendigerweise unterschiedliche Positionen ab: Mia beginnt als entschiedene Anhängerin der METHODE, um sich dann im Laufe der Handlung - insbesondere nachdem der Justizirrtum im Fall Moritz Holl publik geworden ist entschieden zu ihrem Bruder zu bekennen. ${ }^{1174}$ (Diese Kompromisslosigkeit im Angedenken des Bruder lässt Mia als Wiedergängerin der Antigone des Sophokles erscheinen. ${ }^{1175}$ ) Anders als Moritz erkennt Mia jedoch die Irrationalität dieser kompromisslosen Positionierung an: „Ab heute [...] macht sein Name jede Vernunft unmöglich. Ab heute tue ich alles aus Liebe und frei von Furcht.“ (CD,

1172 Vgl. Koellner: Data, Love, and Bodies, S. 410.

1173 Schmidt: Die Erfindung der Realität, S. 268. Zur Mehrdeutigkeit des Untertitels siehe auch Müller-Dietz: Strafrecht im Zukunftsstaat?, S. $433 \mathrm{f}$.

1174 Im Interview benennt Zeh den Reifungsprozess der Figuren als eine Konstante ihrer Werke: „Das Menschenbild, das ich baue, ist positiv, auch wenn man das auf den ersten Blick nicht denkt, weil die Bücher immer düster sind und vom Scheitern erzählen. Aber am Ende kommen die Figuren immer an einen Punkt, an dem sie überhaupt erst fit geworden sind, um Verantwortung für sich selbst zu übernehmen. Für mich sind die Enden der Bücher erst der Anfang für das ,Leben` der Figur. Im Grunde erzähle ich immer Vorgeschichten.“ (Zeh: Der Unterschied zwischen Realität und Fiktion ist marginal, S. 20).

1175 Vgl. Schmidt: Die Erfindung der Realität, S. 268. 
174) Mias Absage an die Vernunft und das entschiedene Bekenntnis zu ihrem Bruder erwachsen dabei nicht so sehr aus der Überzeugungskraft von Moritz' Argumenten, sondern aus der Fundamentalopposition gegenüber einem System, welches durch Vertuschung von Justizirrtümern, Verleumdungen und Folter zumindest in den Augen der Protagonistin jeglicher Legitimität verlustig gegangen ist. Gleichzeitig scheint offenkundig, dass mit einer solchen Totalopposition „kein Staat zu machen ist“: ${ }^{1176}$ Als Staats- und Herrschaftstheorie taugt Kramers nihilistischer Pragmatismus weit besser als Mias trotziger Idealismus (vgl. CD, $233 \mathrm{f}$.). Zwar entzieht Mia mit einem politischen Pamphlet, das ihre Kritik am herrschenden System thesenartig zusammenfasst, „einer Gesellschaft das Vertrauen, die aus Menschen besteht und trotzdem auf der Angst vor dem Menschlichen gründet.“ (CD, 186) Positive Systemalternativen oder spezifische Handlungsvorschläge formuliert sie jedoch nicht - und unterscheidet sich damit deutlich von ihrem realweltlichen Komplement Juli Zeh, die sich immer wieder mit konkreten Reformvorschlägen oder „Aufruf[en] zum Handeln“ öffentlich zu Wort gemeldet hat. ${ }^{1177}$

Anstatt auf die Errichtung eines neuen Systems hinzuarbeiten, klagt Mia lediglich das Recht ein, das herrschende System immer wieder in Frage stellen zu dürfen. Als der Justizirrtum im Fall Moritz Holls publik wird, richtet ein Reporter die Frage an sie:

„Frau Holl, kann die METHODE, wenn sie mit derartigen Fehlern behaftet ist, noch als legitim gelten?“

„Diese Frage“, sagt Mia, „werde ich nicht beantworten. [...] Aber ich werde diese Frage stellen [...] Immer wieder.“

(CD, 170)

Anstelle einer Botschaft oder klaren Handlungsanweisung tritt somit die Forderung, das Verhältnis von Staat und Individuum immer wieder aufs Neue kritisch zu prüfen und neu auszuhandeln. Die Stellung des „Dazwischen“ (CD, 144), welche aus der Perspektive der herrschenden Ordnung als die Position der „Hexe“, der Außenseiterin oder des „Gefährders“ (CD, 253) erscheint, erweist sich in Corpus Delicti somit letztlich als der eigentliche Ort des Politischen.

1176 Diese Formulierung ist übernommen aus Juli Zeh: Die Diktatur der Demokraten. Warum ohne Recht kein Staat zu machen ist. Hamburg 2012. Es handelt sich hierbei um eine Sachbuch-Bearbeitung von Zehs Doktorarbeit.

1177 Trojanow / Zeh: Angriff auf die Freiheit, S. 136. Auch McCalmont und Maierhofer beobachten einen unterschiedlichen Grad an Explizitheit der politischen Forderung zwischen Corpus Delicti und Angriff auf die Freiheit: „Where the play and novel leave the audience disturbed by an imaginary example set in the future and provoke thinking about such issues, the essay raises not just questions but spells out explicit warnings, directly addressing the reader." (McCalmont / Maierhofer: Juli Zeh's Copus Delicti (2009), S. 382) Zu Zehs Reformvorschlägen siehe auch Herminghouse: The Young Author as Public Intellectual. 


\subsubsection{Schlussbetrachtung: die ,Botschaft' der Literatur}

Achtung bitte, wir unterbrechen diesen Text für eine wichtige Durchsage: Dies ist keine Science-fiction. Wir wiederholen: Keine Science-fiction. Dies ist nicht 1984 in Ozeanien, sondern die Gegenwart in der Bundesrepublik. ${ }^{1178}$

Diese Sätze stammen zwar aus der Einleitung des Sachbuchs Angriff auf die Freiheit. Mit geringen Modifikationen ließen sie sich aber auch auf Zehs Roman Corpus Delicti beziehen. Die dystopisch-futurische Diegese des Textes verweist, so will es die Autorin und so legt es die transfiktionale Verweisstruktur des Romans nahe, letztlich auf die reale Welt der Leser. Indem Corpus Delicti, wie Birte Giesler schreibt, „unter der Oberfläche Schlummerndes in die Zukunft projizier[t]“1179, werden Gegenwartstendenzen, gerade in ihrer kontrafaktischen Variation und dystopischen Zuspitzung, zur Kenntlichkeit entstellt. Der Roman kritisiert eine biopolitisch motivierte Absolutsetzung der Gesundheit als zentralem gesellschaftlichen und politischen Wert, eine protonormalistische Einschränkung individueller Freiheiten und die mediale Hexenjagd auf politisch Andersdenkende.

Den Vereindeutigungsversuchen von Totalitarismus, Fanatismus und Radikalismus setzt der Roman die Dialektik der Freiheit entgegen. So behauptet Moritz Holl (der Sache nach wenig konsistent mit seiner sonstigen radikalindividualistischen Gesinnung, möglicherweise aber gerade deshalb als Stimme der Werkintention): „Man muss flackern. Subjektiv, objektiv. Subjektiv, objektiv. Anpassung, Widerstand. An, aus. Der freie Mensch gleicht einer defekten Lampe.“ (CD, 149) Gerade eine solche dynamische Aushandlung von Freiheitsgrenzen enthält der totalitäre Staat in Corpus Delicti seinen Bürgern vor, indem er ihnen - in Erfüllung eines vermeintlich natürlichen Vernunftgebots - die Abwägung konfligierender Interessen abnimmt respektive vorenthält. Ein Staat jedoch, der in jedem Falle besser zu wissen glaubt, was das Richtige für seine Bürger ist, als diese Bürger selbst, endet, so die These des Romans, unausweichlich in der Diktatur.

Die Praktiken der journalistischen Diffamierung politischer Gegner, der flächendeckenden Überwachung der Bürger und der Folter werden im Roman zweifelsohne negativ bewertet. Gleichwohl wird man kaum die These vertreten können, der Roman rede einem naiven Anti-Etatismus das Wort. ${ }^{1180}$ Dass Staats-

1178 Trojanow / Zeh: Angriff auf die Freiheit, S. 11.

1179 Giesler: Zeitgenössisches Drama als rückwärts gekehrte Dystopie in Juli Zehs Corpus Delicti, S. 288.

1180 So die These des ebenso wertungsfreudigen wie philologisch (und auch weltanschaulich) fragwürdigen Artikels von Henk de Berg: Mia gegen den Rest der Welt. Zu Juli Zehs Corpus Delicti. In: Kalina Kupczyńska / Artur Pełka (Hg.): Repräsentationen des Ethischen. Festschrift für Joanna Jabłkowska. Frankfurt a. M. 2013, S. 25-48. 
macht und Justiz prinzipiell sinnvolle und notwendige Einrichtungen sind, stellt die „Dichterjuristin“" ${ }^{1181}$ Juli Zeh auch in ihren fiktionalen Werken nicht in Frage. Corpus Delicti bringt nicht simplifizierend das freie Individuum in Opposition zum grundsätzlich oppressiven Staat. ${ }^{1182}$ Vielmehr macht der Roman deutlich, dass die jeweiligen Abhängigkeiten zwischen beiden immer wieder aufs Neue ausgehandelt werden müssen. Eine Interpretation von Corpus Delicti, die nicht einfach nach leicht zitablen Botschaften sucht, sondern die der literarischen Inszenierung des Politischen im Text nachspürt, wird eine Komplexität in der Behandlung politischer und gesellschaftlicher Fragestellungen entdecken, wie sie mit der in der Forschung verschiedentlich behaupteten Eindeutigkeit des politischen Appells nur bedingt vereinbar ist und wie sie auch noch über das von der Autorin selbst prätendierte ,altmodische[...], aufklärerische[...] Unterfangen“ mit dem sprichwörtlichen „erhobenen Zeigefinger“ hinausgeht. ${ }^{1183}$ Corpus Delicti ruft seine Leser nicht dazu auf, den Prozess der politischen Bewusstwerdung seiner Protagonistin schlicht zu imitieren. Trotz der engen Gegenwartsanbindung ihrer einzelnen Elemente weist die futurische Diegese in ihrer Gesamtheit letztlich eine zu geringe Schnittmenge mit der realen Welt der Leser auf, als dass diese aus dem Roman unmittelbare Handlungsanweisungen für die Gegenwart ableiten könnten (umso weniger, als die tendenziell positiv gezeichneten Figuren des Romans - Mia und Moritz Holl, der Strafverteidiger Rosentreter, der verhalten kritische Journalist Würmer - letztlich allesamt scheitern). Auch entwirft Mia keine positive Systemalternative, sondern formuliert lediglich Kritik am gegebenen System. Das politische Projekt des Romans besteht mithin nicht in der Formulierung einer politischen Agenda, sondern vielmehr in dem Versuch, Problemzusammenhänge der Gegenwart herauszustellen und die Leserschaft zu einer Positionierung gegenüber diesen Problemzusammenhängen aufzurufen. ${ }^{1184}$ Letztlich besteht, so die Formulierung der Autorin, die Wirkabsicht

1181 Vgl. Wagner: Aufklärer der Gegenwart, S. 64-69.

1182 Zwar ruft Mia am Ende ihres Strafprozesses tatsächlich zum Staatsumsturz auf: „Brennt das Land nieder [...] Reißt das Gebäude ein. Holt die Guillotine aus dem Keller, tötet Hunderttausende! [...] Tötet oder schweigt. Alles andere ist Theater.“ (CD, 258) Indem Mia allerdings direkt im Anschluss die Frage stellt: „Wo ist mein Applaus?“ (CD, 258), macht sie deutlich, dass sie hier gerade nicht ihre tatsächliche Meinung zum Ausdruck bringt, sondern vielmehr die Logik des Schauprozesses, zu dessen Opfer sie wird, öffentlich auszustellen sucht. Mias Aufruf zu Mord und Totschlag sollte entsprechend weder als die finale politische Positionierung der Figur noch auch der Autorin angesehen werden.

1183 Zeh: „Ich weiß, dass ich permanent über Moral schreibe.“, S. 58.

1184 Hierin stimmt meine Deutung von Corpus Delicti mit derjenigen von Carrie Smith-Prei überein: „Zeh's use of ambivalence toward delineating or placing a value on moral or ethical rights and wrongs challenges readers to engage critically with her texts and politically with 
von Corpus Delicti darin, „Leute dazu einzuladen, eine Haltung zu entwickeln. “1185 Nicht Mia Holls eigene - durchaus ambivalente - Positionen zu den Themen Gesundheit, Freiheit und Staat sollen also übernommen werden. Vielmehr empfiehlt Juli Zeh den Lesern des Romans ihren je eigenen ,Prozess der politischen Bewusstwerdung an.

Für eine Diskussion der dystopischen Kontrafaktik erweist sich Corpus Delicti in mehrfacher Hinsicht als aufschlussreich: Zehs Roman demonstriert eindrücklich, in welch ein komplexes Abhängigkeits- und Bedingungsgefüge die für das dystopische Genre konstitutive futurische Diegese, die Referenzstruktur der Kontrafaktik und der auktoriale Anspruch, eine politische Botschaft zu formulieren, miteinander treten können. So greift Zeh bei der Behandlung des Themas Hexenverfolgung auf frühneuzeitliches Faktenmaterial zurück, welches dann in der fiktionalen Welt des Romans kontrafaktisch variiert wird (Mia Holl etwa erscheint als Wiedergängerin der historischen Maria Holl und wird in der fiktiven Zukunftsdiegese des Romans zum Opfer einer medialen ,Hexenjagd‘). Ziel dieser Faktenvariationen ist es, gesellschaftliche und politische Entwicklungen der eigenen Gegenwart, welche in Form präziser Anspielungen eindeutig zu identifizieren sind (so etwa der Anspielung auf den Folterskandal von Abu Ghuraib), kritisch zu kommentieren und zugleich auf deren exemplarisch-überzeitliche Bedeutung hinzuweisen. Die erzählpragmatische Funktion der Verweisstruktur von Corpus Delicti ergibt sich somit gerade aus dem Umstand, dass gar keine allzu große Abwandlung nötig ist, um die mittelalterlichen Verfolgungs- und Folterpraktiken auf ein fiktives Zukunftsszenario zu übertragen, welches sich dann seinerseits wiederum erschreckend plausibel auf die Gegenwart der Leser beziehen lässt. Gewissermaßen in Form eines schlüssigen Paradoxes nutzt Zeh in ihrem Roman die kontrafaktischen Faktenvariationen, um zu betonen, dass die historische Abfolge der politischen Systeme nicht immer und nicht notwendigerweise radikale Veränderungen zeitigt, sondern dass die Geschichte im Gegenteil eine beunruhigende Tendenz zur Wiederholung des Immergleichen aufweist. ${ }^{1186}$ Diese Tendenz zur Wiederho-

their contexts. This is true also of Corpus Delicti [...]. Through her literary and extraliterary engagement, Zeh asks readers to evaluate critically the political implications of the eradication of private freedoms, as the public and the private are increasingly and problematically conflated.“ (Smith-Prei: Relevant Utopian Realism, S. 121f.).

1185 Zeh: „Ich weiß, dass ich permanent über Moral schreibe.“, S. $58 \mathrm{f}$.

1186 Schotte und Vorbeck-Heyn fassen diese komplexe zeitliche Verweisstruktur des Romans in der kondensierten Formulierung, der Roman nutze „[d]ie Gefahren manipulativer Mechanismen für eine Gesellschaft [...], um zu zeigen, wie gegenwärtig die Vergangenheit in ihren Argumentationsfiguren und Praktiken künftig zu bleiben droht.“ (Schotte / Vorbeck-Heyn: Die Zukunft unserer Gesellschaft liegt in ihrer Vergangenheit, S. 129). 
lung wird in der Struktur des Romans selbst aufgegriffen, wenn das Urteil gegen Mia Holl bereits am Anfang des Textes wiedergegeben wird, sodass die Erzählinstanz am Ende des Gerichtsprozesses konstatieren kann: „Siehe oben. Siehe wieder und wieder und immer wieder, siehe früh im Jahrhundert und spät im Jahrhundert und mitten im Jahrhundert - oben.“ (CD, 259)

Für eine kontrafaktische Deutung von Corpus Delicti von Interesse ist ferner die signifikante Teilidentität zwischen der Autorin Juli Zeh und der Figur Mia Holl. Indem Zeh die eigene Person zum Ausgangsmaterial einer kontrafaktischautofiktionalen Variation macht, hebt sie den schwierigen Stand des politisch Andersdenkenden - etwa des öffentlichen Intellektuellen - in der Gegenwartsgesellschaft hervor, wie sie es auch in ihren nicht-fiktionalen Texten verschiedentlich getan hat. So bemerkt Zeh etwa in einem Interview aus dem zeitlichen Umfeld der Publikation von Corpus Delicti:

Das Feuilleton jammert und klagt seit Jahren, es rufen circa alle vier Wochen Leute an, die im Radio oder anderen Medien einen Bericht über die Frage machen wollen: Warum gibt es keine Intellektuellen mehr, die sich politisch äußern? Ich sage zu denen immer: Die gibt es schon, aber die paar, die es gibt, macht ihr die ganze Zeit fertig! $!^{1187}$

Dass auch Juli Zeh sich in ihrer Rolle als ,public intellectual' immer wieder medialen Anfeindungen ausgesetzt sah und sieht - ebenso wie andere engagierte Intellektuelle vor ihr, etwa Günter Grass, Heinrich Böll oder Peter Handke (wenn auch im Falle Zehs vermutlich verschärft durch die weitgehende „Delegitimierung von Engagement in Literatur und Literaturwissenschaft der neunziger Jahre“"1188) -, lässt sich anhand der medialen Reaktionen auf Zehs öffentliche Äußerungen leicht nachvollziehen. ${ }^{1189}$ Durch die Teilidentifikation der eigenen Person mit der ,Hexe‘ Mia Holl weist Zeh auf die Problematik, wenn nicht gar auf die Gefahren einer Diffamierung von Personen des öffentlichen Lebens hin, die zu politischen und gesellschaftlichen Entwicklungen kritisch Stellung beziehen. Indem die Position der ,Hexe“ innerhalb des Romans zur eigentlich politischen Position aufgewertet wird, verteidigt Zeh die Legitimität und Notwendigkeit einer Infragestellung des Status quo durch Schriftsteller und öffentliche Intellektuelle - auch und gerade in Zeiten, in denen derartige Zweifel am herrschenden System wenig opportun erscheinen. Zeh nutzt somit in Corpus Delicti Formen der autofiktionalen Kontrafaktik, um einen kritischen Meta-Kommentar zur Funktionsweise des öffentlichen Diskurses zu formulieren.

1187 Zeh: Der Unterschied zwischen Realität und Fiktion ist marginal, S. $57 \mathrm{f}$.

1188 Peitsch: ,Vereinigungsfolgen'.

1189 Aufgearbeitet ist die öffentliche Rezeption der politischen Autorin Zeh bei Wagner: Aufklärer der Gegenwart, S. 70-101. 
Schließlich demonstriert Zeh mit ihrem Engagement für bestimme politische Themen in ganz unterschiedlichen Textformen und Äußerungskontexten, dass Autoren, die sich mit ihren Werken (variierend) auf realweltliches Faktenmaterial beziehen wollen, nicht notwendigerweise von einem fixen Kenntnisstand der Leserschaft ausgehen müssen. ${ }^{1190}$ Anstatt auf vorgegebene Inhalte der Durchschnittsenzyklopädie zu spekulieren, können Autoren eben auch aktiv auf die Erweiterung dieser Enzyklopädie hinarbeiten, etwa in faktualen Medien wie Interviews, Sachbüchern oder Zeitungsartikeln. Indem Juli Zeh außerhalb ihrer literarischen Werke auf Themen - respektive ,Fakten“ - wie Gesundheitswahn, Überwachung und Bürgerrechtsverletzungen aufmerksam macht, bereitete sie ihre Leser gleichsam vor für eine adäquate Rezeption derjenigen fiktionalen Werke, für die eine Identifikation und interpretatorische Berücksichtigung diese Fakten besonders relevant ist. Als engagierte Autorin agiert Zeh mithin sowohl diesseits wie jenseits der Fakten. Ihre faktualen Kommentare $\mathrm{zu}$ politischen Themen und die literarische Gestaltung derselben Themen stehen dabei jedoch nicht einfach unvermittelt nebeneinander. ${ }^{1191}$ Zumindest im Falle von Zehs dystopischer Kontrafaktik treten realweltliche Faktenpräsentation und fiktionale Faktenvariation in ein synergetisches Verhältnis der wechselseitigen Erhellung und politisch-normativen Verstärkung.

1190 Siehe Kapitel 4.7. Markierung.

1191 Schönfellner zufolge kann bei Juli Zeh „von einer Wechselwirkung zwischen literarischem und nicht-fiktionalem Schreiben gesprochen werden“ (Schönfellner: Erzählerische Distanzierung und scheinbare Zukünftigkeit, S. 543). 